\title{
Distance and routing labeling schemes for non-positively curved plane graphs *⿻一𠃋
}

\author{
Victor Chepoi $^{\mathrm{a}, *}$, Feodor F. Dragan ${ }^{\mathrm{b}}$, Yann Vaxès ${ }^{\mathrm{a}}$ \\ a Laboratoire d'Informatique Fondamentale de Marseille, Université de la Méditerranée, \\ Faculté des Sciences de Luminy, F-13288 Marseille cedex 9, France \\ b Department of Computer Science, Kent State University, Kent, OH 44242, USA
}

Received 7 October 2003

Available online 15 September 2004

In memory of Chiril Prisăcaru

\begin{abstract}
Distance labeling schemes are schemes that label the vertices of a graph with short labels in such a way that the distance between any two vertices $u$ and $v$ can be determined efficiently (e.g., in constant or logarithmic time) by merely inspecting the labels of $u$ and $v$, without using any other information. Similarly, routing labeling schemes are schemes that label the vertices of a graph with short labels in such a way that given the label of a source vertex and the label of a destination, it is possible to compute efficiently (e.g., in constant or logarithmic time) the port number of the edge from the source that heads in the direction of the destination. In this paper we show that the three major classes of nonpositively curved plane graphs enjoy such distance and routing labeling schemes using $O\left(\log ^{2} n\right)$ bit labels on $n$-vertex graphs. In constructing these labeling schemes interesting metric properties of those graphs are employed.
\end{abstract}

(C) 2004 Elsevier Inc. All rights reserved.

Keywords: Distance labeling scheme; Routing labeling scheme; Planar graphs; Efficient algorithms

\footnotetext{
Part of this work was done while the second author was visiting the Laboratoire d'Informatique Fondamentale de Marseille. This author thanks the Université de la Méditerranée for support.

* Corresponding author.

E-mail addresses: chepoi@lif.univ-mrs.fr (V. Chepoi), dragan@cs.kent.edu (F.F. Dragan), vaxes@lif.univ-mrs.fr (Y. Vaxès).
}

0196-6774/\$ - see front matter ㄷ 2004 Elsevier Inc. All rights reserved. 


\section{Introduction}

Traditional graph representations are global in nature, and require users to have access to data on the entire graph topology in order to derive useful information, even if the sought piece of information is very local, and pertains to only few vertices.

In contrast, the notion of adjacency labeling scheme, introduced in [5,6] involves using more localized labeling schemes for graphs. The idea is to assign each vertex $v$ a label $L(v)$ in a way that will allow one to infer the adjacency of two vertices directly from their labels, without using any additional information. Obviously, labels of unrestricted size can be used to encode any desired information. However, efficiency considerations dictate the use of relatively short labels (say, of length polylogarithmic in $n$ ), which nevertheless allow us to deduce adjacencies efficiently (say, in constant time). The feasibility of such efficient adjacency labeling schemes is explored in [19,31]. Interest in this natural idea was recently revived by the observation that in addition to adjacency labeling schemes, it may be possible to device similar schemes for capturing distance, connectivity, flow and other information $[11,20,26,27,33]$.

\subsection{Distance labeling schemes}

The notion of distance labeling schemes was first introduced in [27], where also the relevance of distance labeling schemes in the context of communication networks was pointed out. A graph family $\mathcal{D}$ is said to have an $l(n)$ distance labeling scheme if there is a function $L$ labeling the vertices of each $n$-vertex graph in $\mathcal{D}$ with distinct labels of up to $l(n)$ bits, and there exists an algorithm, called distance decoder, that given two labels $L(v), L(u)$ of two vertices $v, u$ in a graph from $\mathcal{D}$, decides the distance between $v$ and $u$ in time polynomial in the length of the given labels. Note that the algorithm is not given any additional information, other that the two labels, regarding the graph from which the vertices were taken.

As noticed in [19], a class of $2^{\Omega\left(n^{1+\varepsilon}\right)} n$-vertex graphs, must use adjacency labels (and thus distance labels) whose total length is $\Omega\left(n^{1+\varepsilon}\right)$. Hence, at least one label must be of $\Omega\left(n^{\varepsilon}\right)$ bits. Specifically, for the class of all unweighted graphs, any distance labeling scheme must label some $n$-vertex graphs with labels of size $\Omega(n)$. This raises the natural question of whether more efficient labeling schemes can be constructed for special graph classes.

A distance labeling scheme for trees that uses only $O\left(\log ^{2} n\right)$ bit labels and a constant time distance decoder has been given in [26]. ${ }^{1}$ This result is complemented by a lower bound proven in [17], showing that $\Omega\left(\log ^{2} n\right)$ bit labels are necessary for the class of all trees. The scheme developed for trees was later extended in $[17,20]$ to other graph classes with "well-behaved" separators; $O\left(\log ^{2} n\right)$ distance labeling schemes were presented for interval graphs, permutation graphs, distance-hereditary graphs and all graphs of bounded tree width, while an $O(\sqrt{n} \log n)$ distance labeling scheme was presented for all planar

\footnotetext{
1 [26] claims to have only $O(\log n)$ time distance decoder, but in fact it is not hard to make that decoder to run in constant time.
} 
graphs. Recently, authors of [16] improved the bound on the label size given in [17] for interval graphs by a $\log n$ factor. For the class of planar graphs only a lower bound of $\Omega\left(n^{1 / 3}\right)$ on the label size is known. This leaves an intriguing polynomial gap between upper and lower bounds on the label size.

\subsection{Routing labeling schemes}

Routing is one of the basic tasks that a distributed network of processors must be able to perform. A routing scheme is a mechanism that can deliver packets of information from any vertex of the network to any other vertex. Each packet has a header containing the address of the destination, and in some cases, some additional information that can be used to guide the routing of this message. Each processor in the network has a routing daemon running on it which is endowed with a local routing table. This daemon receives packets of information and has to decide, based on this table and on the packets headers only, whether these packets have already reached their destination, and if not, how to forward them towards their destination. One aims at routing along short paths. This problem can be approached via localized techniques based on labeling schemes [28].

Following [28], a family $\Re$ of graphs is said to have an $l(n)$ routing labeling scheme if there exist a function $L$, labeling the vertices of each $n$-vertex graph in $\Re$ with distinct labels of up to $l(n)$ bits, and an efficient algorithm, called the routing decision, that given the label of a current vertex $v$ and the label of the destination vertex (the header of the packet), decides in time polynomial in the length of the given labels and using only those two labels, whether this packet has already reached its destination, and if not, to which neighbor of $v$ to forward the packet. Thus, the goal is, for a family $\Re$ of graphs, to find routing labeling schemes employing shortest (or nearly shortest) paths and using relatively short labels and fast routing decision.

There are many results on routing schemes for particular graph classes, including complete graphs, grids (alias meshes), hypercubes, complete bipartite graphs, unit interval and interval graphs, trees and 2-trees, rings, tori, unit circular-arc graphs, outerplanar graphs, and squaregraphs (see $[1,9,13,14,21,25,30]$ ). All those graph families admit routing schemes with $O(d \log n)$ labels and $O(\log d)$ routing decision (where $d$ is the maximum degree of a vertex). These results follow from the existence of so called interval routing schemes for those graphs. Observe that in interval routing schemes the local memory requirement increases with the degree of the vertex. Routing labeling schemes aim overcoming the problem of large degree vertices. In [33] and independently in [11], an optimal (in view of [12]) routing labeling scheme for trees of arbitrary maximum degree is described that assigns each vertex of an $n$-vertex tree a $(1+o(1)) \log _{2} n$-bit label and has a constant time routing decision. For planar graphs, a routing labeling scheme which uses $8 n+o(n)$ bits per vertex is developed in [15].

\subsection{Our contribution}

In this note we design distance and routing labeling schemes for three natural classes of planar graphs introduced in $[23,24]$ and further investigated in $[3,4,29,32]$ and the 
references cited therein. These are the basic classes of planar graphs of non-positive combinatorial curvature:

(i) the plane graphs with all inner faces of length at least 4 (the length of a face is the number of edges of its bounding cycle) and with all inner vertices of degree at least 4 (called the $(4,4)$-graphs),

(ii) the plane graphs with all inner faces of length at least 3 and all inner vertices of degree at least 6 (called the $(3,6)$-graphs), and

(iii) the plane graphs with all inner faces of length at least 6 and all inner vertices of degree at least 3 (called the $(6,3)$-graphs).

Based on geometric properties of these graph classes, we design for them labeling schemes with labels of size $O\left(\log ^{2} n\right)$ bits and a constant time distance decoder and routing decision.

The paper is organized as follows. The next section presents the main definitions and notions. Section 3 describes the general lines of the method used for distance queries and routing in all three classes of graphs. In Section 4 we establish the principal distance properties of $(4,4)-,(3,6)-$, and $(6,3)$-graphs used in these schemes. In Section 5 we give a detailed presentation of the labeling and routing schemes for $(4,4)$ - and $(3,6)$-graphs, while the case of $(6,3)$-graphs is treated in the technical report [8] of this paper.

\section{Preliminaries}

All graphs $G=(V, E)$ occurring in this paper are undirected, unweighted, connected, $n$-vertex plane graphs, i.e., planar graphs embedded on the plane. The distance $d(u, v):=$ $d_{G}(u, v)$ between two vertices $u$ and $v$ is the length of a shortest $(u, v)$-path, and the interval $I(u, v)$ between $u$ and $v$ consists of all vertices on shortest $(u, v)$-paths, that is, $I(u, v)=\{x \in V: d(u, x)+d(x, v)=d(u, v)\}$. An induced subgraph of $G$ (or the corresponding vertex set $A$ ) is called convex if it includes the interval between any of its vertices. For a set $S \subseteq V$ and a vertex $x$ of $G$, the projection $\operatorname{Pr}(x, S)$ of $x$ on $S$ consists of all vertices $v \in S$ such that $I(v, x) \cap S=\{v\}$. Notice that $I(s, x) \cap \operatorname{Pr}(x, S) \neq \emptyset$ for any vertex $s \in S$. Extending the notion of a gated set from [10], we call a set $S \subseteq V$ quasigated if for every vertex $x \notin S$ the projection $\operatorname{Pr}(x, S)$ consists of one or two adjacent vertices of $S$ (called the gates of $x$ ).

For a plane graph $G$, let $\partial G$ be the cycle (actually, closed walk) bounding the outer face of $G$ oriented counterclockwise and let $G^{*}$ be the geometric dual of $G$ (in which vertices are defined only for inner faces of $G$ ). We call the paths of $G^{*}$ dual paths. Notice that the class of $(4,4)$-graphs is self-dual in the sense that the geometric dual of a $(4,4)$-graph is again a $(4,4)$-graph, while the classes of $(3,6)$ - and $(6,3)$-graphs are mutually dual. Two neighbors $x, y$ of a vertex $v$ of $G$ are called consecutive if $v, x, y$ belong to a common inner face of $G$. Following [4,22,24] and the references therein, we introduce now the curvature function of a plane graph $G$. Assume that each inner face with $k$ sides of $G$ is viewed as a regular $k$-gon in Euclidean plane with side length 1. For a vertex $v$ of $G$, let $\alpha(v)$ denote the sum of the corner angles of the regular polygons containing the vertex $v$. If $v$ is an inner vertex of $G$, denote the curvature at $v$ to be $\kappa(v)=2 \pi-\alpha(v)$, i.e., it is defined as 
the $2 \pi$-angle-defect of the flat polygons meeting at $v$. When $v$ is a vertex in the boundary $\partial G$, define the turning angle at $v$ to be $\tau(v)=\pi-\alpha(v)$. A vertex $v \in \partial G$ with $\tau(v)>0$ is called a corner of $G$. The following Lyndon's curvature theorem [24] is a discrete version of the Gauss-Bonnet theorem and holds for all plane graphs:

$$
\sum_{v \in V-\partial G} \kappa(v)+\sum_{v \in \partial G} \tau(v)=2 \pi .
$$

A plane graph $G$ has non-positive curvature if $\kappa(v) \leqslant 0$ for every inner vertex $v$ of $G$. It can be easily shown that the plane graphs of each of the types $(4,4),(3,6)$, and $(6,3)$ have non-positive curvature, and from this perspective they have been investigated in a number of papers; cf. for example $[4,23,24]$. From the Gauss-Bonnet formula it follows that a plane graph of non-positive curvature has at least 3 corners. In Section 4, we will further specify this property for each type of those graphs.

For an edge $u v$ of a graph $G$, define the following partition of the vertex set $V$ :

$$
\begin{aligned}
& W(u, v)=\{x \in V: d(x, u)<d(x, v)\}, \\
& W(v, u)=\{x \in V: d(x, v)<d(x, u)\}, \\
& W_{=}(u v)=\{x \in V: d(x, v)=d(x, u)\} .
\end{aligned}
$$

If $G$ is bipartite, then the set $W_{=}(u v)$ is empty. A cut $\{A, B\}$ of $G$ is a partition of the vertex-set $V$ into two parts, and a convex cut is a cut in which the halves $A$ and $B$ are convex. Denote by $E(A, B)$ the set of all edges of $G$ having one end in $A$ and another one in $B$, and say that those edges are crossed (or cut) by $\{A, B\}$. The zone $Z(A, B)$ of the cut $\{A, B\}$ is the family of inner faces of $G$ sharing edges with $E(A, B)$. A zone $Z(A, B)$ is called a strip if the faces of $Z(A, B)$ induce a simple dual path and two faces $F^{\prime}, F^{\prime \prime}$ of $Z(A, B)$ intersect if and only if they share an edge of $E(A, B)$. The union of faces of a strip constitutes a simply connected region of the plane. If $Z(A, B)$ is a strip, then we will use the same notation $Z(A, B)$ for the (plane) subgraph of $G$ induced by the vertices and the edges occurring in the faces of this zone. The inner faces of this graph are exactly the inner faces of $G$ from $Z(A, B)$. For a strip $Z(A, B)$, call the subgraphs induced by $\partial A=Z(A, B) \cap A$ and $\partial B=Z(A, B) \cap B$ the border lines of the cut $\{A, B\}$.

We continue with the definition of alternating cuts introduced and investigated in [7,29]. Two edges $e^{\prime}=\left(u^{\prime}, v^{\prime}\right)$ and $e^{\prime \prime}=\left(u^{\prime \prime}, v^{\prime \prime}\right)$ on a common inner face $F$ of $G$ are called opposite in $F$ if $d_{F}\left(u^{\prime}, u^{\prime \prime}\right)=d_{F}\left(v^{\prime}, v^{\prime \prime}\right)$ and equals the diameter of the cycle $F$. If $F$ is an even face, then each edge has a unique opposite edge, otherwise, if $F$ is an odd face, then every edge $e \in F$ has two opposite edges $e^{+}$and $e^{-}$sharing a common vertex. In the latter case, if $F$ is oriented clockwise, for $e$ we distinguish the left opposite edge $e^{+}$ and the right opposite edge $e^{-}$. If every face of $Z(A, B)$ is crossed by a cut $\{A, B\}$ in two opposite edges, then we say that $\{A, B\}$ is an opposite cut of $G$. We say that an opposite cut $\{A, B\}$ is straight on an even face $F \in Z(A, B)$ and that it makes a turn on an odd face $F \in Z(A, B)$. The turn is left or right depending which of the pairs $\left\{e, e^{+}\right\}$or $\left\{e, e^{-}\right\}$it crosses. An opposite cut $\{A, B\}$ of a plane graph $G$ is alternating if the turns on it alternate. Denote by $\mathcal{A C}(G)$ the collection of all alternating cuts of $G$, where every cut which never has to turn is counted twice.

Finally, for a graph $G=(V, E)$ and a vertex $x$, let $F(x)=\sum_{v \in V} d(x, v)$. Any vertex minimizing the function $F$ is called a median vertex of the graph $G$. Notice the following 
simple but important property of the function $F$ : if uv is an edge of $G$, then $F(v)-F(u)=$ $|W(u, v)|-|W(v, u)|$. From this we immediately conclude that if $v$ is a median vertex of $G$, then $|W(u, v)| \leqslant|V| / 2$ for any neighbor $u$ of $v$.

\section{General method}

Our distance and routing labeling schemes are based on geometric properties of alternating cuts of $(4,4)-,(3,6)$-, and $(6,3)$-graphs $G$. Some of them have been already proven in $[3,29]$ in order to establish the scale 2 embedding of these graphs into hypercubes. First, we prove that any alternating cut $\{A, B\}$ of $G$ is convex, moreover its border lines are convex paths (thus the zone of every such cut is a strip sharing two edges with $\partial G$ ). Then, in case of $(4,4)$-graphs, we show that the zones of alternating cuts are quasigated. For $(3,6)$-graphs, we show that the projections $\operatorname{Pr}(x, Z(A, B))$ on zones of alternating cuts are convex paths whose vertices have the same distance to $x$ (the structure of projections on zones of $(6,3)$-graphs is given in [8]). In all cases, the projection $\operatorname{Pr}(x, Z(A, B))$ can be compactly represented by the end vertices and the type of this projection. For example, for $(4,4)$-graphs, at vertex $x$ we will keep the gate(s) on $Z(A, B)$ and the distance from $x$ to the projection. For $(3,6)$-graphs, we will keep the end vertices of the convex path $\operatorname{Pr}(x, Z(A, B))$ and the distance from $x$ to the projection. For routing messages from $x$, the following property of $\operatorname{Pr}(x, Z(A, B))$ is crucial: we show that either there is a neighbor of $x$ one step closer to $Z(A, B)$ whose projection on this zone coincides with that of $x$ or there exist two neighbors of $x$ one step closer to the zone and whose projections on $Z(A, B)$ cover the projection of $x$. We will keep at $x$ the information about such neighbors and use it in the routing decision. So, in all cases we need only $O(\log n)$ bits to store at $x$ the entire information about the relative position of $x$ with respect to the zone $Z(A, B)$. Therefore, we can report in constant time the distance between two vertices $x \in A$ and $y \in B$ using only the information related to $Z(A, B)$ stored at $x$ and $y$ (for this we design also an $O(1)$-time algorithm for computing the distance between two projections on $Z(A, B))$. However, we need more information in order to compute the distances between two vertices of $A$ or two vertices of $B$.

To compute the distances or a routing shortest path between arbitrary two vertices of $G$, we describe a distributed data structure which at each vertex $x \in V$ keeps the projections of $x$ on the zones of only $O\left(\log _{2} n\right)$ alternating cuts of $G$. For this, let $v$ be a median vertex of $G$ and let $u_{0}, u_{1}, \ldots, u_{k-1}$ be its neighbors in counterclockwise order around $v$, according to the embedding of $G$ in the plane. (We may assume without loss of generality that $v$ is an inner vertex of $G$, otherwise we can add a constant number of vertices and faces around $v$ to transform it into an inner vertex and obtain a graph of the same type.) Every edge $v u_{i}$ is crossed by two alternating cuts $\left\{A_{i}^{\prime}, B_{i}^{\prime}\right\}$ and $\left\{A_{i}^{\prime \prime}, B_{i}^{\prime \prime}\right\}$ such that $v \in A_{i}^{\prime} \cap A_{i}^{\prime \prime}$ and $u_{i} \in B_{i}^{\prime} \cap B_{i}^{\prime \prime}$. Let us orient the cuts $\left\{A_{i}^{\prime}, B_{i}^{\prime}\right\}$ and $\left\{A_{i}^{\prime \prime}, B_{i}^{\prime \prime}\right\}$ such that $v$ is on the left border line. In this case, we will denote by $\left\{A_{i}^{\prime}, B_{i}^{\prime}\right\}$ that cut from the two alternating cuts separating $u_{i}$ and $v$, such that the last turn before $u_{i} v$ is on the right (if it exists) and the next turn after $u_{i} v$ is on the left (if it exists). If none of these two turns exists, then $\left\{A_{i}^{\prime}, B_{i}^{\prime}\right\}$ and $\left\{A_{i}^{\prime \prime}, B_{i}^{\prime \prime}\right\}$ coincide. For each vertex $u_{i}$, set $C_{v}\left(u_{i}\right)=B_{i}^{\prime} \cap A_{i+1(\bmod k)}^{\prime}$ and call $C_{v}\left(u_{i}\right)$ a cone with apex $u_{i}$. Every cone is convex as the intersection of two 
convex sets. We show that $C_{v}\left(u_{i}\right) \subseteq W\left(u_{i}, v\right)$, yielding $\left|C_{v}\left(u_{i}\right)\right| \leqslant n / 2$ because $v$ is a median vertex. Furthermore, we establish that the cones $C_{v}\left(u_{0}\right), \ldots, C_{v}\left(u_{k-1}\right)$ together with the vertex $v$ form a partition of the vertex-set of $G$. To report the distance or a routing path between two query vertices $x$ and $y$ efficiently, yet another property of the partition $C_{v}\left(u_{0}\right) \cup \cdots \cup C_{v}\left(u_{k-1}\right) \cup\{v\}$ is significant. We call two neighbors $u_{i}, u_{j}$ of $v p$ consecutive and their cones $C_{v}\left(u_{i}\right), C_{v}\left(u_{j}\right)$ p-neighboring if $\min \{|i-j|, k-|i-j|\}=p$. Let $x \in C_{v}\left(u_{i}\right)$ and $y \in C_{v}\left(u_{j}\right)$. We show that if $C_{v}\left(u_{i}\right)$ and $C_{v}\left(u_{j}\right)$ are not $p$-neighboring for $p \leqslant 2$, then $d(x, y)=d(x, v)+d(v, y)$. Therefore, in order to report distance between two vertices $x$ and $y$ in different cones, we have to keep their distances to the median vertex $v$, the projections on and the distances to the 1-neighboring and 2-neighboring cones, more precisely on/to the zones separating the respective cones. Finally, if $x$ and $y$ belong to the same cone $C_{v}\left(u_{i}\right)$, then the distance $d(x, y)$ can be retrieved by recursively decomposing the (convex) subgraph $G_{i}$ induced by $C_{v}\left(u_{i}\right)$. Routing between $x$ and $y$ can be performed by converting the distance labeling scheme in the following way. To route a message from $x$ to $y$ lying in different cones, additional to distances, we have to store in the label of $x$ the output port number of the first edge on a shortest path from $x$ to $v$ and the output port number of the first edge on a shortest path from $x$ to each of the two end vertices of the projection of $x$ on the 1-neighboring as well as 2-neighboring cones, or more precisely on the zones separating the respective cones. If $x$ is its own projection on the zone between $x$ and $y$, we consider the relative position of $x$ and the projection of $y$ on the zone to decide in constant time via which edge the message should be sent. Finally, if $x$ and $y$ belong to the same cone $C_{i}(v)$, then, again, the routing can be done by recursively decomposing the subgraph $G_{i}$ induced by this cone.

All these facts suggest the necessity of building a decomposition tree $T(G)$ of $G$, which can be constructed in the following way. Find a median vertex $v$ of $G$ and the cones $C_{v}\left(u_{0}\right), \ldots, C_{v}\left(u_{k-1}\right)$ with apices at the neighbors of $v$. Let $G_{i}$ be the subgraph of $G$ induced by $C_{v}\left(u_{i}\right)$. For each $G_{i}$ construct a decomposition tree $T\left(G_{i}\right)$ recursively and build $T(G)$ by taking pair $(G, v)$ to be the root and connecting the root of each tree $T\left(G_{i}\right)$ as a child of $(G, v)$. It is easy to see that a decomposition tree $T(G)$ of a graph $G$ with $n$ vertices has depth at most $\log _{2} n$ and can be constructed in $O\left(n^{2} \log n\right)$ time. Indeed, in each level of recursion we need to find median vertices of current subgraphs and to construct the corresponding cones. Also, since the graph sizes are reduced by a factor $1 / 2$, the recursion depth is $O(\log n)$.

For tree $T(G)$ we need a labeling scheme for depths of nearest common ancestors (NCA-depth labeling scheme). In [27] such a scheme with $O\left(\log ^{2} n\right)$ bit labels but with $O(\log n)$ query time was presented for any tree with $n$ nodes. One can use here the fact that $T(G)$ has the $O(\log n)$ depth and get constant query time in this case. To do this one can simply translate the technique of Harel and Tarjan [18] to a labeling scheme. Note that whenever they access global information, it is associated with an ancestor in a tree. Since the depth of our tree is $O(\log n)$, one can copy this ancestor information down to each descendant and get the desired label of $O\left(\log ^{2} n\right)$ bits. Thus, tree $T(G)$ can be preprocessed in $O(n \log n)$ time for depths of nearest common ancestors. This preprocessing step creates for $T(G)$ an NCA-depth labeling scheme with $O\left(\log ^{2} n\right)$ bit labels and constant query time. For each vertex $x$ of a graph $G$, let $S(x)$ be the deepest node of $T(G)$ containing $x$ and $A_{x}$ be the label of $S(x)$ in the NCA-depth labeling scheme. Let also $S_{0}, S_{1}, \ldots, S_{h}$ be 
the nodes of the path of $T(G)$ from the root $(G, v)$ (which is $S_{0}$ ) to the node $S(x)=S_{h}$. Clearly, $h \leqslant \log _{2} n$.

In our distance (or routing) labeling scheme, vertex $x$ will keep in its label $L(x)$ the string $A_{x}$ and $O\left(\log _{2} n\right)$ strings of $O(\log n)$ bits, one for each node $S_{i}(i \in\{0, \ldots, h\})$. The string for $S_{i}=\left(G_{i}, v_{i}\right)$ will contain the distance (or routing) and projection information obtained during the decomposition of a subgraph $G_{i}$ using its median vertex $v_{i}$. To report the distance between vertices $x$ and $y$ of $G$ (or to route a message from $x$ to $y$ ), we can do the following. First, using strings $A_{x}$ and $A_{y}$, find the depth in $T(G)$ of the nearest common ancestor $S_{k}=\left(G_{k}, v_{k}\right)$ of $S(x)$ and $S(y)$. Clearly, vertices $x$ and $y$ belong to different cones defined by $v_{k}$ in $G_{k}$. Therefore, one can apply the method described above to compute $d_{G}(x, y)$ (or the port number of an edge incident to $x$ which heads in the direction of $y$ ) in constant time using only the strings in $L(x)$ and $L(y)$ which correspond to the node $S_{k}$ of $T(G)$.

Using this general method, the rest of this paper is devoted to establishing the following main result:

Theorem 3.1. The family of graphs of type $(4,4),(3,6)$, and $(6,3)$ with at most $n$ vertices admits distance and routing labeling schemes with labels of size $O\left(\log ^{2} n\right)$ bits and a constant time distance decoder and routing decision. Moreover, the schemes are constructable in time $O\left(n^{2} \log n\right)$.

\section{Geometry of $(4,4)$ - and $(6,3)$-graphs}

Here we establish the metric and structural properties of $(4,4)-,(3,6)-$, and $(6,3)$ graphs used in the distance and routing labeling schemes outlined in Section 3 and described in details in Section 5. In the following results, unless specified, $G$ is a plane graph of one of those types.

\subsection{Alternating cuts}

We start with a result first established in [23] for the three classes of plane graphs in question and later extended to all plane graphs of non-positive curvature in [4].

Lemma 4.1. [4,23] For each vertex $x$ of a plane graph $G$ of non-positive curvature, all vertices at maximum distance from $x$ are located on the outer face $\partial G$.

As we noticed in Section 2, every plane graph $G$ of non-positive curvature has at least 3 corners. If $G$ is a $(4,4)$-graph, then every corner of $G$ is a vertex of degree 2 , and from the Gauss-Bonnet formula we conclude that in fact such a graph $G$ must contain at least 4 corners. In a similar way, one concludes that a $(3,6)$-graph either contains exactly 3 corners which are vertices of degree 2 incident to inner faces of length 3 or at least 4 corners. In the latter case, the corners are either vertices of degree 2 or vertices of degree 3 incident to two inner faces, one of length 3 and another of length at most 5. Finally, in a $(6,3)$-graph all corners are vertices of degree 2 and $G$ contains at least 6 corners. The following sharper 
version of this result established in [3] will be of more use: $\partial G$ contains at least 6 edges whose end vertices are corners; we call them corner edges (this again follows easily from the Gauss-Bonnet formula).

We continue with the properties of alternating cuts of $G$. Several lemmata have been proven in the unpublished manuscript [3] for $(3,6)$ - and $(6,3)$-graphs. For $(4,4)$-graphs, the analogies of some of those results have been established in [29]. For the sake of completeness we provide all results with proof (see also [8] for specific properties of $(6,3)$ graphs).

According to [7], every edge $e$ of a plane graph $G$ is crossed by at most two alternating cuts, which can be constructed using the following algorithm. We go from $e$ in two directions (or in only one direction if $e$ belongs to the outer face of $G$ ) until we arrive at odd faces. In this movement we go straight through even faces. Now, suppose that $F$ and $D$ are the first odd faces which occur when moving in opposite directions. Then in one cut we make left turn on $F$ and right turn on $D$, and in another cut we make right turn on $F$ and left turn on $D$ (see Fig. 3 below for an illustration). After that we have only to alternate the directions when passing through odd faces of $G$. Namely, if say our last turn in one cut was to the left, then coming to the next odd face this cut turns to the right, and conversely. Let $E^{\prime}(e)$ and $E^{\prime \prime}(e)$ be the two (not necessarily distinct) groups of edges which we cross in this movement. It is noticed in [7] that for any alternating cut $\{A, B\}$ which cuts the edge $e$ either $E(A, B)=E^{\prime}(e)$ or $E(A, B)=E^{\prime \prime}(e)$ holds. Therefore, if we will show that for every edge $e$ of $G$ the sets $E^{\prime}(e)$ and $E^{\prime \prime}(e)$ define cuts, then these cuts necessarily will be alternating, thus showing that every edge of $G$ is crossed by exactly two cuts from $\mathcal{A C}(G)$

Let $Z^{\prime}(e)$ and $Z^{\prime \prime}(e)$ denote the union of inner faces of $G$ sharing edges with $E^{\prime}(e)$ and $E^{\prime \prime}(e)$, respectively. By construction, both $Z^{\prime}(e)$ and $Z^{\prime \prime}(e)$ generate dual paths. Moreover, if the algorithm never crosses the same face twice, then these dual paths are simple and the end vertices of each of them are inner faces of $G$ sharing edges with $\partial G$. We assert that if $Z^{\prime}(e)$ and $Z^{\prime \prime}(e)$ are simple dual paths, then $E^{\prime}(e)$ and $E^{\prime \prime}(e)$ define cuts of $G$, namely that the end vertices $x$ and $y$ of $e$ belong to distinct connected components of the graphs $G^{\prime}=\left(V, E-E^{\prime}(e)\right)$ and $G^{\prime \prime}=\left(V, E-E^{\prime \prime}(e)\right)$. Suppose, by way of contradiction, that $x$ and $y$ can be connected in $G^{\prime}$ by a (simple) path $P$. Consider the closed region $R$ of the plane bounded by the cycle formed by $P$ and $e$. Let $F^{\prime}$ be the inner face of $G$ incident to $e$ and located in $R$. Running the algorithm for $E^{\prime}(e)$, we will first cross $F^{\prime}$, and then some other inner faces of $G$ located in $R$. Since no edge of $P$ is crossed, we will get stuck in $R$, contrary to the assumption that $Z^{\prime}(e)$ is a simple path of $G^{*}$. Instead of proving that $Z^{\prime}(e)$ and $Z^{\prime \prime}(e)$ are simple dual paths, we will establish a slightly stronger result:

Lemma 4.2. [3,29] For every edge e of $G, Z^{\prime}(e)$ and $Z^{\prime \prime}(e)$ are strips. In particular, every edge e of $G$ is crossed by two cuts from $\mathcal{A C}(G)$.

Proof. Suppose, by way of contradiction, that $Z^{\prime}(e)$ is not a strip. Then either $Z^{\prime}(e)$ is not a simple path and we obtain the configurations one and four from Fig. 1, or two faces of $Z^{\prime}(e)$ intersect in a single vertex and we obtain the configurations two and three depicted in Fig. 1. In the first three cases of this figure, consider the subgraph $H$ of $G$ induced by all vertices lying in the bounded region $R$. Obviously $H$ has the same type as $G$. If $G$ is a 

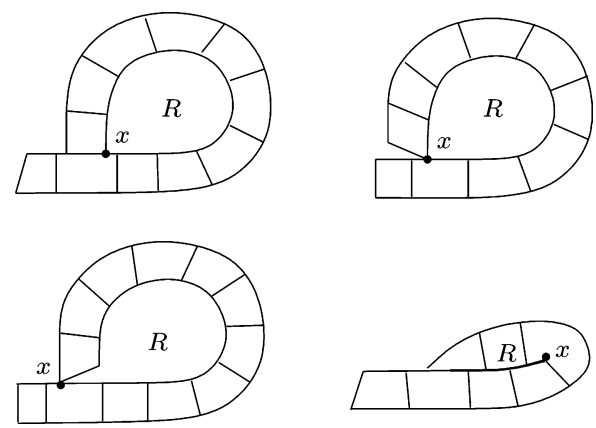

Fig. 1. To the proof of Lemma 4.2.

$(4,4)$-graph, then all vertices of $\partial H$ except one must have degree larger that two, otherwise we get an inner vertex of degree 2 or 3 in $G$. However this contradicts the fact that $H$ must have at least 4 corners. Analogously, if $G$ is a $(6,3)$-graph, $H$ cannot contain any corner edge. If $G$ is a $(3,6)$-graph, any corner of $H$ different from $x$ will be an inner vertex of $G$ of degree at most 5, again leading to a contradiction. In the fourth case, the region $R$ degenerates into a path, and one of the end vertices of this path (namely, the vertex $x$ ) is an inner vertex of $G$ having degree 2 , a contradiction.

This shows that $Z^{\prime}(e)$ and $Z^{\prime \prime}(e)$ are strips, hence $E^{\prime}(e)$ and $E^{\prime \prime}(e)$ define two alternating cuts of $G$. These cuts coincide if and only if $E^{\prime}(e)=E^{\prime \prime}(e)$, nevertheless this cut is counted twice in $\mathcal{A C}(G)$. Since any alternating cut crossing the edge $e$ is obtained in this way, we conclude that $e$ is crossed by exactly two cuts from $\mathcal{A C}(G)$.

Lemma 4.3. [3] The border lines of an alternating cut are convex paths. In particular, the alternating cuts of $G$ and their zones are convex.

Proof. From Lemma 4.2 we conclude that $\partial A$ and $\partial B$ are paths. Therefore, it suffices to establish that they are convex. Assume the contrary and among alternating cuts with nonconvex border lines pick an alternating cut $\{A, B\}$ such that $\partial A$ contains a closest pair of vertices $x$ and $y$ which can be connected by a shortest $(x, y)$-path $P$ such that $P \cap \partial A=\{x, y\}$. Since the lengths of the subpaths of $\partial A$ and $\partial B$, comprised between the end vertices of two edges of $E(A, B)$, differ by at most 1 (because the cut $\{A, B\}$ is alternating), necessarily the whole path $P$ must belong to the set $A$ : if $P$ is not contained in $A$, then we can replace $x, y \in \partial A$ with a pair of vertices of $\partial B \cap P$, contrary to our choice. Let $z$ be a neighbor of $x$ on the path $P$. Consider the alternating cuts $\left\{A^{\prime}, B^{\prime}\right\}$ and $\left\{A^{\prime \prime}, B^{\prime \prime}\right\}$ of $\mathcal{A C}(G)$ which cross the edge $x z$. If one of these cuts, say $\left\{A^{\prime}, B^{\prime}\right\}$, crosses another edge $x^{\prime} y^{\prime}$ of $P$, where $z, y^{\prime} \in A^{\prime}$, then by replacing $x, y \in \partial A$ with the pair $z, y^{\prime} \in \partial A^{\prime}$, we will get a contradiction with the choice of $x, y$. Thus, we may assume that both these alternating cuts separate some adjacent vertices $u, v$ of the path $\partial A$, say $x, u \in \partial A^{\prime}$ and $z, v \in \partial B^{\prime}$. We will obtain one of the situations depicted in Fig. 2. In the first case, let $H$ be the subgraph of $G$ comprised in the region $R$. Let $t$ be the closest to $u$ common vertex of $\partial A$ and $\partial A^{\prime}$. If $G$ is a $(4,4)$-graph, then $H$ may contain only two corners $t$ and $u$. If $G$ is a $(6,3)$-graph, then $H$ may contain at most four corner edges which are all incident to $t$ 

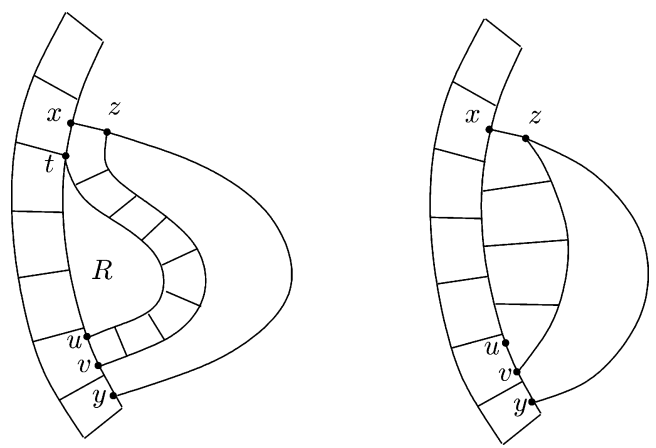

Fig. 2. To the proof of Lemma 4.3.

or to $u$. Finally, if $G$ is a $(3,6)$-graph, then any corner $w$ of $H$ other than $t$ and $u$ will be an inner vertex of $G$ having degree at most 5: if, say $w \in \partial A^{\prime}$, then $w$ has at most 4 neighbors in the zone $Z\left(A^{\prime}, B^{\prime}\right)$ and yet another neighbor located in the interior of the region $R$. This contradicts the fact that $H$ must contain at least three corners. Now consider the second possibility from Fig. 2. If $G$ is a $(4,4)$-graph or a $(6,3)$-graph, then from the definition of an alternating cut one concludes that $u$ cannot have other neighbors in $\partial B^{\prime}$ except $v$. Since $u$ can have at most one neighbor in $\partial B, u$ is an inner vertex of $G$ of degree 2 or 3 , which is impossible if $G$ is of type $(4,4)$. But, if $u$ has degree 3 , then necessarily the inner face of $Z(A, B)$ containing the edge $u v$ is either of length 4 or 5 , yielding a contradiction if $G$ is of type $(6,3)$. Finally, if $G$ is of type $(3,6)$, since $\partial A$ and $\partial A^{\prime}$ share the subpath between $u$ and $x$, one can easily deduce that $u$ is an inner vertex of $G$ of degree at most 5, a contradiction. This shows that the border lines of alternating cuts of $G$ are convex paths, thus the alternating cuts of $G$ and their zones are convex, too.

Since every edge of $G$ is crossed by exactly two alternating cuts from $\mathcal{A C}(G)$ and these cuts are convex by previous result, an observation of [2] implies that any pair of vertices $x, y$ of $G$ can be separated by exactly $2 d(x, y)$ cuts of $\mathcal{A C}(G)$.

Let $e=x y$ be an edge of $G$ and let $\left\{A^{\prime}, B^{\prime}\right\}$ and $\left\{A^{\prime \prime}, B^{\prime \prime}\right\}$ be the (not necessarily distinct) alternating cuts crossing $e$, where $x \in A^{\prime} \cap A^{\prime \prime}$ and $y \in B^{\prime} \cap B^{\prime \prime}$. Let $E\left(A^{\prime}, B^{\prime}\right)=$ $E^{\prime}(e)$ and $E\left(A^{\prime \prime}, B^{\prime \prime}\right)=E^{\prime \prime}(e)$. We will establish now a relation between $A^{\prime}, B^{\prime}, A^{\prime \prime}, B^{\prime \prime}$ and the sets $W(x, y), W(y, x), W_{=}(x y)$ (the third set here may be empty). By removing the edges of $E^{\prime}(e) \cup E^{\prime \prime}(e)$ from $G$ but leaving their end vertices, we get a graph whose connected components are induced by the pairwise intersections $A^{\prime} \cap A^{\prime \prime}, B^{\prime} \cap B^{\prime \prime}, A^{\prime} \cap B^{\prime \prime}$, and $A^{\prime \prime} \cap B^{\prime}$. We assert that these convex sets coincide with $W(x, y), W(y, x)$ and the connected components of $W_{=}(x y)$. First notice that from the definition of alternating cuts and the convexity of their border lines it follows that $Z=Z^{\prime}(e) \cap Z^{\prime \prime}(e)$ consists of one or several faces constituting a strip. Notice that each of the end faces of $Z$ either shares an edge with the outer face of $G$ or is an odd face. Denote by $F$ and $D$ these odd faces if they exist (in fact, $F$ and $D$ are the faces defined in the algorithm of construction of these alternating cuts). Notice that all other faces of $Z$ have even length. Let $u v$ and $w z$ be the first edges of $F$ and $D$ cut by $\left\{A^{\prime}, B^{\prime}\right\}$ and $\left\{A^{\prime \prime}, B^{\prime \prime}\right\}$ while moving from $x y$ towards these faces, and assume that $u, w \in A^{\prime} \cap A^{\prime \prime}$ and $v, z \in B^{\prime} \cap B^{\prime \prime}$. Let $p r^{\prime}$ and $p r^{\prime \prime}$ be the opposite 


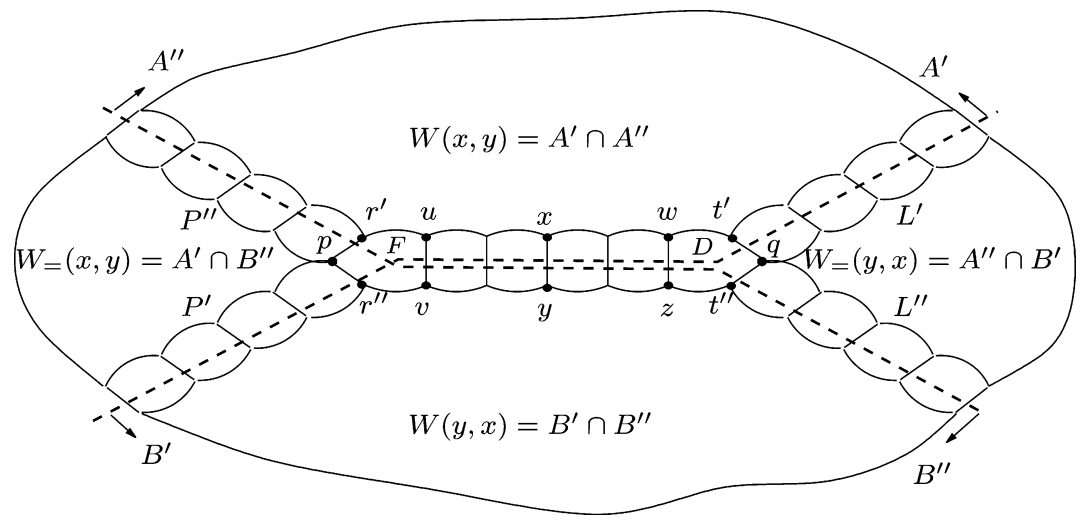

Fig. 3. The relationship between $A^{\prime}, B^{\prime}, A^{\prime \prime}, B^{\prime \prime}$ and $W(x, y), W(y, x), W_{=}(x y)$.

to $u v$ edges of $F$ and let $q t^{\prime}$ and $q t^{\prime \prime}$ be the opposite to $w z$ edges of $D$. Assume that the cut $\left\{A^{\prime}, B^{\prime}\right\}$ makes turns at the edges $p r^{\prime \prime}$ and $q t^{\prime}$ such that $p, t^{\prime} \in \partial A^{\prime}$ and $r^{\prime \prime}, q \in \partial B^{\prime}$. Then, the cut $\left\{A^{\prime \prime}, B^{\prime \prime}\right\}$ makes turns at the edges $p r^{\prime}$ and $q t^{\prime \prime}$ such that $r^{\prime}, q \in \partial A^{\prime \prime}$ and $p, t^{\prime \prime} \in \partial B^{\prime \prime}$; see Fig. 3 for an illustration. Since the zones $Z^{\prime}(e), Z^{\prime \prime}(e)$ and their border lines are convex, we conclude that all vertices of $\partial A^{\prime}$ are closer to $x$ than to $y$, all vertices of $\partial B^{\prime \prime}$ are closer to $y$ than to $x$, while the vertices of the subpaths $L^{\prime}, L^{\prime \prime}$ of $\partial B^{\prime}$ and $\partial A^{\prime \prime}$, comprised between $q$ and their end vertices of $\partial G$, are equidistant from $x$ and $y$ as well as the vertices of the subpaths $P^{\prime}, P^{\prime \prime}$ of $\partial A^{\prime}$ and $\partial B^{\prime \prime}$, comprised between $p$ and their end vertices of $\partial G$. Now, if we pick a vertex $z$ in $B^{\prime} \cap A^{\prime \prime}$, then any shortest path between $z$ and $x$ or $y$ must cross one of the paths $L^{\prime}$ or $L^{\prime \prime}$, therefore $z$ is equidistant from $x$ and $y$. Analogously, all vertices of $A^{\prime} \cap B^{\prime \prime}$ are equidistant from $x$ and $y$, while all vertices of $A^{\prime} \cap A^{\prime \prime}$ are closer to $x$ than to $y$ and all vertices of $B^{\prime} \cap B^{\prime \prime}$ are closer to $y$ than to $x$. Since any path connecting vertices from different convex sets $A^{\prime} \cap A^{\prime \prime}, A^{\prime} \cap B^{\prime \prime}, B^{\prime} \cap A^{\prime \prime}, B^{\prime} \cap A^{\prime \prime}$ necessarily employs an edge of $E^{\prime}(e) \cup E^{\prime \prime}(e)$, these sets are the connected components of the graph obtained from $G$ by removing the edges of $E^{\prime}(e) \cup E^{\prime \prime}(e)$. If both $F$ and $D$ do not exist, then the cuts $\left\{A^{\prime}, B^{\prime}\right\}$ and $\left\{A^{\prime \prime}, B^{\prime \prime}\right\}$ coincide, and if only one of the faces $F, D$ exists, then $W_{=}(x y)$ consists of a single convex component. Summarizing, we obtain the following result:

Lemma 4.4. $W(x, y)=A^{\prime} \cap A^{\prime \prime}, W(y, x)=B^{\prime} \cap B^{\prime \prime}$, while $W_{=}(x, y):=B^{\prime} \cap A^{\prime \prime}$ and $W_{=}(y, x):=A^{\prime} \cap B^{\prime \prime}$ constitute a partition of $W_{=}(x y)$ into two (maybe empty) convex subsets.

From the previous discussion we also conclude that every vertex $z^{\prime} \in W_{=}(x, y)$ can be connected to $x$ and $y$ by a shortest path going via $p$. Moreover, $p$ is the furthest from $z^{\prime}$ vertex of $I\left(z^{\prime}, x\right) \cap I\left(z^{\prime}, y\right)$. We call $p$ the apex of $z^{\prime}$ with respect to $x$ and $y$. Analogously, one can define the apex of every vertex $z^{\prime \prime} \in W_{=}(y, x)$ and see that it coincides with the vertex $q$ (see Fig. 3). 


\subsection{Faces}

We continue with some properties of inner faces of $G$.

Lemma 4.5. The intersection of two inner faces of $G$ is empty, a vertex, or an edge.

Proof. Let $F^{\prime}$ and $F^{\prime \prime}$ be two intersecting inner faces. Then $F^{\prime} \cap F^{\prime \prime}$ cannot contain paths of length 2 , because all inner vertices must have degree at least 3 . Thus, $F^{\prime} \cap F^{\prime \prime}$ consists of a sequence of isolated vertices and edges. Let $R$ be the region bounded by two paths of $F^{\prime}$ and $F^{\prime \prime}$, respectively, comprised between two consecutive non-adjacent common vertices. The subgraph $H$ of $G$ comprised in $R$ has the same type as $G$ but does not contain vertices of degree 2 at all. This leads to a contradiction for the case of $(4,4)$ - and $(6,3)$-graphs. If $G$ is a $(3,6)$-graph, any corner of degree 3 of $H$ is an inner vertex of degree 3 or 4 of $G$, which is impossible. This establishes our assertion.

Lemma 4.6. Every inner face $F$ of $G$ is convex.

Proof. Assume, by way of contradiction, that two (necessarily non adjacent) vertices $u, v$ of $F$ can be connected by a shortest path $P$ outside $F$, i.e., $P \cap F=\{u, v\}$. From Lemma 4.3 we conclude that $u$ and $v$ will be separated by any alternating cut $\{A, B\}$ which crosses the face $F$. Let $\{A, B\}$ cross $F$ along the edges $u^{\prime} v^{\prime}$ and $u^{\prime \prime} v^{\prime \prime}$, where $u^{\prime}, u^{\prime \prime} \in A$, $v^{\prime}, v^{\prime \prime} \in B$ and suppose, without loss of generality, that the edge $u^{\prime} v^{\prime}$ is located in the interior of the region of the plane bounded by $P$ and the subpath of $F$ comprised between $u$ and $v$ and passing via the edge $u^{\prime \prime} v^{\prime \prime}$. Moreover, $\{A, B\}$ cuts the path $P$ in some edge $x y$, where $x \in A$ and $y \in B$. Then $u^{\prime}, u^{\prime \prime}, x \in \partial A$ and $v^{\prime}, v^{\prime \prime}, y \in \partial B$. By Lemma 4.3, $\partial A$ and $\partial B$ are convex paths of the graph $G$. Thus, one of the vertices $u^{\prime}, u^{\prime \prime}, x$ is located in $\partial A$ between other two. Notice also that the vertex $u$ is between $u^{\prime}$ and $u^{\prime \prime}$, therefore it also belongs to $\partial A$. Since the subpath of $P$ between $u$ and $x$ is a shortest path, it also belongs to $\partial A$. Therefore $u=u^{\prime}$, otherwise the vertex $u$ of $\partial A$ will have three neighbors in $\partial A$. Analogously, one concludes that $v=v^{\prime}$, i.e., $u$ and $v$ are adjacent, contrary to their choice.

\section{Lemma 4.7. Every inner face $F$ of $G$ is quasigated.}

Proof. Suppose there exist vertices of $G$ whose projections on $F$ contain non-adjacent vertices and among such triplets select the vertices $x \notin F, u, v \in \operatorname{Pr}(x, F)$ minimizing the distance sum $d(x, u)+d(u, v)+d(v, x)$. Pick shortest $(u, x)$ - and $(v, x)$-paths $P^{\prime}$ and $P^{\prime \prime}$. Let $Q^{\prime}$ and $Q^{\prime \prime}$ be the subpaths of $F$ comprised between $u$ and $v$, and assume, without loss of generality, that $Q^{\prime}$ is located in the interior of the region bounded by the closed walk $P^{\prime} \cup P^{\prime \prime} \cup Q^{\prime \prime}$. Let $w$ be the neighbor of $u$ in $Q^{\prime}$. Then $d(x, w) \geqslant d(x, u)$, because $u \in \operatorname{Pr}(x, F)$. As we noticed above, there exist exactly $2 d(u, x)$ cuts of $\mathcal{A C}(G)$ separating $u$ and $x$ and exactly $2 d(w, x)$ cuts of $\mathcal{A C}(G)$ separating $w$ and $x$. Every alternating cut separating the vertex $w$ from $x$ and $v$ also separates $u$ from $x$. Analogously, every alternating cut separating $x$ from $v$ and $w$ also separates $x$ from $u$. If both alternating cuts separating $u$ and $w$ also separate $u$ and $x$, then we will obtain that $2 d(u, x)>2 d(w, x)$, which is impossible. Therefore there exists a cut $\{A, B\} \in \mathcal{A C}(G)$ such that $u, x \in A$ and $w, v \in B$. This 
cut separates two adjacent vertices $p, q \in P^{\prime \prime}$ and two adjacent vertices $u^{\prime}, w^{\prime} \in Q^{\prime \prime}$, where $u^{\prime}, p \in A$ and $v^{\prime}, q \in B$. Notice that $u^{\prime}, u, p \in \partial A$ and $v^{\prime}, w, q \in \partial B$. Since $\partial B$ is convex by Lemma 4.3, the vertices $v$ and $w$ lie on this path. Therefore, $w \in I(v, q) \subset I(v, x)$, contrary to the assumption that $I(x, v) \cap F=\{v\}$. This establishes that the vertices of $\operatorname{Pr}(x, F)$ are pairwise adjacent. Since the graphs of types $(4,4)$ and $(6,3)$ are triangle-free, we immediately obtain that in this case $\operatorname{Pr}(x, F)$ consists of one or two vertices. If $G$ is of type $(3,6)$, however $|\operatorname{Pr}(x, F)|>2$, then $F$ is 3-cycle $(u, v, w)$ whose vertices have the same distance to $x$. One of these vertices, say $u$, is located in the region $R$ bounded by the edge $v w$ and two shortest paths connecting $v, w$ with $x$. Consider the subgraph (of type $(3,6))$ of $G$ induced by the vertices lying inside $R$ or on the boundary of $R$. By Lemma 4.1, all inner vertices of this graph must be closer to $x$ than $v$ and $w$, which is not the case for $u$.

Lemma 4.8. Given three vertices $x, y, z$ such that $d(x, z)=d(y, z)$ and $d(x, y)=1$, either $x, y, z$ belong to a common odd face or there exists an even face $F_{0}$ such that the edges $x y$ and $z^{\prime} z^{\prime \prime}$ are opposite in $F_{0}$, where $\operatorname{Pr}\left(z, F_{0}\right)=\left\{z^{\prime}, z^{\prime \prime}\right\}$.

Proof. Consider the partition of $G$ provided by Lemma 4.4, and let say $z \in W_{=}(x, y)$. If in Fig. 3 the edge $x y$ belongs to the face $F$, then $F$ is the required odd face, otherwise $F_{0}$ is the even face of $Z$ incident to $x y$ comprised between $x y$ and $F$.

\subsection{Intervals}

The following property of intervals will be of much use.

Lemma 4.9. The vertex $x$ has at most two (consecutive) neighbors in the interval $I(x, y)$. If $G$ is a $(6,3)$-graph, then $I(x, y)$ contains at most two vertices at distance 2 from $x$. Moreover, if $x$ has two neighbors and two vertices at distance 2 in $I(x, y)$, then these five vertices belong to a common inner face of $G$.

Proof. We proceed by induction on $d(x, y)$. Pick two neighbors $u, w$ of $x$ in $I(x, y)$ and let $P^{\prime}$ and $P^{\prime \prime}$ be two shortest $(x, y)$-paths passing via $u$ and $w$. Since $x$ is the unique furthest from $y$ vertex of the closed walk $P^{\prime} \cup P^{\prime \prime}$, from Lemma 4.1 we infer that all vertices of $G$, located in the region $R$ bounded by $P^{\prime} \cup P^{\prime \prime}$, are closer to $y$ than the vertex $x$. Therefore, any neighbor $v \in R$ of $x$ also belongs to the interval $I(x, y)$. So, further assume that $I(x, y)$ contains three consecutive neighbors $u, v, w$, such that $x, u, v$ belong to the face $F^{\prime}$ and $x, v, w$ belong to the face $F^{\prime \prime}$ of $G$. Then $F^{\prime} \cap F^{\prime \prime}=\{x, v\}$, by Lemma 4.5. If $F^{\prime}$ and $F^{\prime \prime}$ have length $\geqslant 4$, let $v^{\prime}$ and $v^{\prime \prime}$ be the (different) neighbors of $v$ in the faces $F^{\prime}$ and $F^{\prime \prime}$. Since $v^{\prime}, v^{\prime \prime} \in I(v, y)$ in view of Lemma 4.7, by the induction assumption, we conclude that $v, v^{\prime}$, and $v^{\prime \prime}$ belong to a common inner face $F$ of $G$. As a consequence, we infer that $v$ is an inner vertex of $G$ of degree 3 , leading to a contradiction if $G$ is of type $(4,4)$ or $(3,6)$. If $F^{\prime}, F^{\prime \prime}$ have length 3 each (i.e., $G$ has the type $\left.(3,6)\right)$, then the edges $u v$ and $v w$ belong to two other inner faces $D^{\prime}$ and $D^{\prime \prime}$. Let $v^{\prime}$ and $v^{\prime \prime}$ be the neighbors of $v$ in $D^{\prime}$ and $D^{\prime \prime}$. Since $v^{\prime}, v^{\prime \prime} \in I(v, y)$ by Lemma 4.8, the vertices $v, v^{\prime}, v^{\prime \prime}$ belong to a common face $D$, and we conclude that $v$ has degree 5 if $v^{\prime} \neq v^{\prime \prime}$ and degree 4 if $v^{\prime}=v^{\prime \prime}$. The case 
when $G$ is of type $(3,6)$ and only one of the faces $F^{\prime}$ and $F^{\prime \prime}$ has length 3 is analogous. On the other hand, if $G$ is of type $(6,3)$, then $v$ has in $I(v, y)$ four vertices at distance 2, in contradiction to the induction hypothesis. Thus, in all cases, $x$ may have maximum two consecutive neighbors in $I(x, y)$. To complete the proof, it remains to establish that if $G$ is of type $(6,3)$, then $I(x, y)$ contains at most two vertices at distance 2 from $x$. Let $u$ and $v$ be the neighbors of $x$ in $I(x, y)$, and let $F$ be the inner face of $G$ passing via $x, u$, and $v$. Let $u^{\prime}$ and $v^{\prime}$ be the neighbors of $u$ and $v$ in $F$, and suppose, by way of contradiction, that $v$ has yet another neighbor $v^{\prime \prime}$ in $I(v, y) \subset I(x, y)$. The vertices $v^{\prime}, v, v^{\prime \prime}$ belong to a common inner face $F^{\prime}$ of $G$. As $F \cap F^{\prime}=\left\{v, v^{\prime}\right\}$ and the faces $F$ and $F^{\prime}$ are quasigated and have at least 6 vertices each, we conclude that the neighbors of $v^{\prime}$ in $F$ and $F^{\prime}$ and the neighbor of $v^{\prime \prime}$ in $F^{\prime}$ are all different and belong to the interval $I(v, y)$, contrary to the induction hypothesis.

\subsection{Projections on zones}

We specify the structure of projections of vertices on zones for each type of graphs.

Lemma 4.10. The zone $Z(A, B)$ of any alternating cut $\{A, B\}$ of a $(4,4)$-graph $G$ is quasigated, i.e., $\operatorname{Pr}(x, Z(A, B))$ consists of one or two adjacent vertices.

Proof. Let $x \in A$ and denote by $a, b$ the end vertices of the path $\partial A$. Let $u$ and $v$ be the vertices of $\operatorname{Pr}(x, Z(A, B))$ closest to $a$ and $b$, respectively. Then $u \in I(a, x)$ and $v \in$ $I(b, x)$. Suppose that $d(x, v) \leqslant d(x, u)$ and call $k=d(x, v)$ the distance of $x$ to $Z(A, B)$. We proceed by induction on $k$. Suppose, by way of contradiction, that $u$ and $v$ are not adjacent. Then, the neighbor $w$ of $v$ in the convex path $I(u, v) \subseteq \partial A$ is different from $u$. Let $y$ be a neighbor of $x$ in $I(x, v)$. Since the distance of $y$ to $Z(A, B)$ is smaller than $k$, by the induction hypothesis $y$ has a quasigate in $Z(A, B)$. Hence, either $\operatorname{Pr}(y, Z(A, B))=\{v\}$ or $\operatorname{Pr}(y, Z(A, B))=\{v, w\}$. Notice that $d(y, w) \geqslant d(y, v)$, otherwise $w \in I(y, v) \subset I(x, v)$, contrary to the fact that $v \in \operatorname{Pr}(x, Z(A, B))$. We will assume that $\operatorname{Pr}(y, Z(A, B))=\{v, w\}$, the other case being similar. Then $w \in I(u, y) \subseteq I(a, y)$. Let $z$ be the neighbor of $u$ in the convex path $I(u, w)$. Then $v, w, y \in W(z, u)$, while $x \notin W(z, u)$ from the choice of $u$. From Lemma 4.4 we infer that one of the alternating cuts (say, $\left\{A^{\prime}, B^{\prime}\right\}$ ), crossing the edge $u z$, also crosses the edge $x y$, say $u, x \in \partial A^{\prime}$ and $z, y \in \partial B^{\prime}$. Lemma 4.3 yields $I(u, x) \subseteq$ $\partial A^{\prime}$ and $I(z, w) \subset I(z, y) \subseteq \partial B^{\prime}$. Hence $u z$ is an inner edge of $E\left(A^{\prime}, B^{\prime}\right)$. Let $F^{\prime}$ and $F^{\prime \prime}$ be the faces of $Z\left(A^{\prime}, B^{\prime}\right)$ sharing $u z$, where $F^{\prime} \in Z(A, B)$. If $z \neq w$, then $F^{\prime \prime}$ contains the edge $z t$ of the path $I(z, w)$ incident to $z$. Since $F^{\prime \prime} \cap F^{\prime}=\{u, z\}$, the edge $z t$ belongs to a face $F \neq F^{\prime}$ of the zone $Z(A, B)$. Since the faces of $G$ have at least 4 edges and the cut $\left\{A^{\prime}, B^{\prime}\right\}$ is alternating, we deduce that $z$ is an inner vertex of degree 3 , a contradiction. Now suppose that $z=w$, i.e., $d(u, v)=2$. In this case, $d(x, u)=d(x, z)=d(x, v)$. Also $d(y, v)=d(y, w)$ because $v, w \in \operatorname{Pr}(y, Z(A, B))$. Let $y_{0}$ be the apex of $y$ with respect to $v, w$, and let $y^{\prime}$ be the neighbor of $y_{0}$ in the convex path $I\left(y_{0}, z\right) \subseteq \partial B^{\prime}$. Then $y^{\prime} \in W(z, v)$ and $y_{0} \notin W(z, v)$, whence, by Lemma 4.4 , there is an alternating cut $\left\{A^{\prime \prime}, B^{\prime \prime}\right\}$ crossing $y_{0} y^{\prime}$ and $v z$, say $y^{\prime}, z \in \partial A^{\prime \prime}$ and $y_{0}, v \in \partial B^{\prime \prime}$. Since $y^{\prime} \in \partial B^{\prime}$ and $G$ has type $(4,4)$, one can easily see that $y^{\prime}$ is an inner vertex of degree at most 3: except its two neighbors in $I(x, z), y^{\prime}$ may have only one other neighbor in $\partial A^{\prime}$. 
For a vertex $x \in A$, we call two vertices $u, v$ of $\operatorname{Pr}(x, Z(A, B)) \subseteq \partial A$ consecutive if the subpath $P$ of $\partial A$ comprised between $u$ and $v$ does not contain other vertices of $\operatorname{Pr}(x, Z(A, B))$.

Lemma 4.11. If $\{A, B\}$ is an alternating cut of $a(3,6)$-graph $G$ and $x \in A$, then $\operatorname{Pr}(x, Z(A, B))$ induces a (convex) subpath of $\partial A$ which consists of vertices having the same distance to $x$.

Proof. $\operatorname{Pr}(x, Z(A, B))$ is a subset of the convex path $\partial A$. To establish the assertion, it suffices to show that two consecutive vertices $u, v$ of $\operatorname{Pr}(x, Z(A, B))$ are adjacent in $G$. Suppose, by way of contradiction, that $u$ and $v$ are not adjacent, and let $P$ be the subpath of $\partial A$ between $u$ and $v$. Then, for any vertex $w \in P \backslash\{u, v\}$, at least one of the vertices $u, v$ belongs to $I(w, x)$. Therefore, there exist two adjacent or coinciding vertices $w^{\prime}, w^{\prime \prime} \in P$ such that $u \in I\left(w^{\prime}, x\right)$ and $v \in I\left(w^{\prime \prime}, x\right)$. If $w^{\prime}=w^{\prime \prime}$, then the neighbors $t^{\prime}$ and $t^{\prime \prime}$ of $w^{\prime}$ in $\partial A$ belong to the interval $I\left(w^{\prime}, x\right)$, therefore $w^{\prime}, t^{\prime}$, and $t^{\prime \prime}$ belong to a common inner face $F$ (by Lemma 4.9). Since $F$ is not in $Z(A, B)$ and $w^{\prime}$ belongs to at most three inner faces of $Z(A, B)$, we deduce that $w^{\prime}$ is an inner vertex of degree at most 4: $w^{\prime}$ has two neighbors in $\partial A$, at most two neighbors in $\partial B$ and no other neighbors, yielding a contradiction.

Now suppose that $w^{\prime}$ and $w^{\prime \prime}$ are adjacent and $w^{\prime} \notin I\left(w^{\prime \prime}, x\right), w^{\prime \prime} \notin I\left(w^{\prime}, x\right)$, whence $d\left(x, w^{\prime}\right)=d\left(x, w^{\prime \prime}\right)$. Notice also that $w^{\prime} \neq u$ or $w^{\prime \prime} \neq v$, say the second. Let $x_{0}$ be the apex of $x$ with respect to $w^{\prime}, w^{\prime \prime}$, and let $x^{\prime} \in I\left(x_{0}, w^{\prime}\right)$ and $x^{\prime \prime} \in I\left(x_{0}, w^{\prime \prime}\right)$ be adjacent to $x_{0}$. Consider the two alternating cuts $\left\{A^{\prime}, B^{\prime}\right\}$ and $\left\{A^{\prime \prime}, B^{\prime \prime}\right\}$ crossing the edge $w^{\prime} w^{\prime \prime}$. Then one of these cuts, say $\left\{A^{\prime}, B^{\prime}\right\}$, will cross the edge $x_{0} x^{\prime}$ and the second one $\left\{A^{\prime \prime}, B^{\prime \prime}\right\}$ will cross the edge $x_{0} x^{\prime \prime}$. Obviously, the cut $\left\{A^{\prime}, B^{\prime}\right\}$ will also cross an edge of every shortest $(x, u)$-path and the cut $\left\{A^{\prime \prime}, B^{\prime \prime}\right\}$ will cross an edge $z^{\prime} z^{\prime \prime}$ of every shortest $(x, v)$ path. Let $x, z^{\prime}, w^{\prime} \in A^{\prime \prime}$ and $z^{\prime \prime}, w^{\prime \prime} \in B^{\prime \prime}$, more precisely $z^{\prime}, w^{\prime} \in \partial A^{\prime \prime}$ and $z^{\prime \prime}, w^{\prime \prime} \in \partial B^{\prime \prime}$. Let also $F$ be the face of $Z(A, B)$ containing the edge $w^{\prime} w^{\prime \prime}$. Since $v \in I\left(w^{\prime \prime}, x\right)$ and $z^{\prime \prime} \in I(v, x)$, we conclude that $v \in I\left(w^{\prime \prime}, z^{\prime \prime}\right) \subseteq \partial B^{\prime}$. Therefore, the vertex $w^{\prime \prime}$ belongs to a face $F \in Z(A, B) \cap Z\left(A^{\prime \prime}, B^{\prime \prime}\right)$, to another face $D$ of $Z\left(A^{\prime \prime}, B^{\prime \prime}\right)$ and maybe to two other faces of $Z(A, B)$. Since $w^{\prime \prime}$ may have only one neighbor in $D \cap \partial A^{\prime \prime}$, except its neighbor in $I\left(w^{\prime \prime}, v\right)$ (this will happen if $D$ has length 3 ), we conclude that $w^{\prime \prime}$ is an inner vertex of $G$ but its degree is 4 or 5 , a contradiction.

In the case of $(6,3)$-graphs, it is shown in [8] that $\operatorname{Pr}(x, Z(A, B))$ is either a vertex, or two adjacent vertices, or a sequence of vertices $u_{1}, u_{2}, \ldots, u_{k}$ of $\partial A$ such that $d\left(u_{i}, u_{i+1}\right)=2$ for all $i=1, \ldots, k-1$; in the latter case, all vertices of $\operatorname{Pr}(x, Z(A, B))$ except maybe the leftmost and/or the rightmost vertices have the same distance to $x$, while one or both end vertices may be one step further from $x$.

Lemma 4.12. Given a vertex $x \in A \backslash \partial A$ and an alternating cut $\{A, B\}$ of $G$, there exist two (not necessarily distinct) neighbors $u_{x}$ and $v_{x}$ of $x$ such that $I(x, w) \cap\left\{u_{x}, v_{x}\right\} \neq \varnothing$ for any vertex $w \in \operatorname{Pr}(x, Z(A, B))$. In particular, $I(x, y) \cap\left\{u_{x}, v_{x}\right\} \neq \emptyset$ for any vertex $y \in B$. Analogously, any vertex $x \in \partial A$ has two neighbors $u_{x}, v_{x}$ in $Z(A, B)$ such that $I(x, y) \cap\left\{u_{x}, v_{x}\right\} \neq \emptyset$ for any vertex $y \in B$. 
Proof. First suppose that $x \in A \backslash \partial A$. The result is obvious if $\operatorname{Pr}(x, Z(A, B))$ consists of one or two vertices (in particular, for $(4,4)$-graphs in view of Lemma 4.10): as $u_{x}$ and $v_{x}$ it suffices to take any neighbors of $x$ on shortest paths connecting $x$ with the vertices from the projection. Analogously, if $x$ contains a neighbor $u_{x} \in I(x, u) \cap I(x, v)$ (where $u$ and $v$ are the end vertices of $\operatorname{Pr}(x, Z(A, B))$ ), then from the properties of projections one concludes that $\operatorname{Pr}\left(u_{x}, Z(A, B)\right)=\operatorname{Pr}(x, Z(A, B))$ and that $u_{x} \in I(x, w)$ for any $w \in \operatorname{Pr}(x, Z(A, B))$. So, further assume that $I(x, u) \cap I(x, v)=\{x\}$, in particular, $d(x, v) \leqslant d\left(u_{x}, v\right)$ for any neighbor $u_{x}$ of $x$ in $I(x, u)$. Let $v^{\prime}$ be the closest to $v$ vertex of $\operatorname{Pr}\left(u_{x}, Z(A, B)\right)$ (i.e., $\left.\operatorname{Pr}\left(u_{x}, Z(A, B)\right) \subseteq I\left(u, v^{\prime}\right)\right)$ and let $t$ be a neighbor of $u_{x}$ in $I\left(u_{x}, v^{\prime}\right) \subseteq I\left(u_{x}, v\right)$. If $d(x, v)<d\left(u_{x}, v\right)$, then denote by $F$ the face containing the vertices $u_{x}, t, x$ (see Lemma 4.9), otherwise if $d(x, v)=d\left(u_{x}, v\right)$, then denote by $F$ the face containing the edge $x u_{x}$ and provided by Lemma 4.8. Let $v_{x}$ be the neighbor of $x$ in $F$ different from $u_{x}$.

First, suppose that $G$ is a graph of type $(3,6)$. By Lemma $4.11, \operatorname{Pr}(x, Z(A, B))=$ $I(u, v)$ and all its vertices have the same distance to $x$. Moreover $\operatorname{Pr}\left(u_{x}, Z(A, B)\right) \subseteq$ $\operatorname{Pr}(x, Z(A, B))$ and every vertex of $\operatorname{Pr}\left(u_{x}, Z(A, B)\right)$ is closer to $u_{x}$ than to $x$, because $u_{x}$ is one step closer to $Z(A, B)$ than $x$. Suppose, by way of contradiction, that $v^{\prime}$ is not adjacent to $v$ (otherwise we are done). Since $v^{\prime} \in I\left(v, u_{x}\right)$ and $d(x, v) \leqslant d\left(u_{x}, v\right)$ we conclude that $d\left(v^{\prime}, v\right)=2$ and $x \in I\left(u_{x}, v\right)$. The face $F$ defined above and passing via $u_{x}, t, x$ will have length $\geqslant 4$, otherwise $x$ and $t$ are adjacent and $u_{x} \notin I\left(x, v^{\prime}\right)$. Since $x, t \in I\left(u_{x}, v\right)$ and $F$ is quasigated, one can easily conclude that $v_{x} \in I(x, v)$. We also assert that $\left\{u_{x}, v_{x}\right\} \cap I(x, w) \neq \emptyset$, where $w$ is the common neighbor of $v^{\prime}$ and $v$. Indeed, the vertex $t$ is closer to $w$ than the vertices $u_{x}$ and $x$, therefore the distance from $w$ to $F$ is at most $d(w, x)-1$, thus $x \notin I(x, w) \cap \operatorname{Pr}(w, F)$ and a shortest path from $x$ to $w$ crossing this intersection will go via $u_{x}$ or $v_{x}$.

\subsection{A distance property}

Let $v$ be an inner vertex of $G$ and let $u_{0}, u_{1}, \ldots, u_{k-1}$ be the neighbors of $v$ labeled counterclockwise. Notice that the sets $W\left(u_{i}, v\right)$ and $W\left(u_{j}, v\right)$ are disjoint unless $u_{i}$ and $u_{j}$ are consecutive or coincide. Indeed, if $z \in W\left(u_{i}, v\right) \cap W\left(u_{j}, v\right)$ and $i \neq j$, then $u_{i}, u_{j} \in$ $I(v, z)$, therefore, by Lemma 4.9, $u_{i}$ and $u_{j}$ are consecutive neighbors of $x$.

Lemma 4.13. If $G$ is a (4,4)-graph (or a $(6,3)$-graph), $x \in W\left(u_{i}, v\right), y \in W\left(u_{j}, v\right)$, and $u_{i}, u_{j}$ are not $p$-consecutive for $p \leqslant 2$, then $v \in I(x, y)$. Analogously, if $G$ is a $(3,6)$ graph, $x \in W\left(u_{i}, v\right), y \in W\left(u_{j}, v\right)$, and $u_{i}, u_{j}$ are not $p$-consecutive for $p \leqslant 3$, then $v \in$ $I(x, y)$.

Proof. First, by induction on $d\left(y, u_{j}\right)$, we will show that if $y \in W\left(u_{j}, v\right)$ and $u_{i}$ and $u_{j}$ are not consecutive in a graph $G$ of type $(4,4)($ or $(6,3))$, then $v \in I\left(u_{i}, y\right)$ (this covers the assertion in the case $\left.x=u_{i}\right)$. An analogous assertion holds for a $(3,6)$-graph provided $u_{i}$ and $u_{j}$ are neither consecutive nor 2-consecutive. The result is obvious if $y=u_{j}$. So, assume $y \neq u_{j}$ and let $y^{\prime}$ be a neighbor of $y$ in $I\left(y, u_{j}\right)$. Since $y^{\prime} \in W\left(u_{j}, v\right)$, the induction hypothesis yields $v \in I\left(u_{i}, y^{\prime}\right)$, therefore, $y^{\prime} \in W\left(v, u_{i}\right)$. If $v \notin I\left(u_{i}, y\right)$, then $y \notin W\left(v, u_{i}\right)$. From Lemma 4.4 there exists an alternating cut $\{A, B\}$ such that $u_{i}, y \in \partial A$ 
and $v, y^{\prime} \in \partial B$. Moreover $u_{j} \in I\left(v, y^{\prime}\right) \subseteq \partial B$. This immediately implies that the vertices $u_{i}, v, u_{j}$ belong to a common face of $Z(A, B)$, which is impossible because $u_{i}$ and $u_{j}$ are not consecutive. For $(3,6)$-graphs either we get the same contradiction, or $u_{i}, v$, and the neighbor $z$ of $u_{i}$ in $\partial A$ constitute a triangular face of $Z(A, B)$, while $v, z$, and $u_{j}$ belong to another face of $Z(A, B)$, from which we infer that $u_{i}$ and $u_{j}$ are 2-consecutive. This contradiction establishes the required inclusion $v \in I\left(u_{i}, y\right)$.

Now we consider the general case $x \neq u_{i}$. We may suppose, without loss of generality, that $I(x, y) \cap W\left(u_{i}, v\right)=\{x\}$, otherwise we can use induction on $d\left(x, u_{i}\right)$. Let $z$ be a neighbor of $x$ in $I(x, y)$. Since $z \notin W\left(u_{i}, v\right)$, by Lemma 4.4, there exists an alternating cut $\{A, B\}$ crossing the edges $u_{i} v$ and $x z$. Let $u_{i}, x \in \partial A$ and $v, z \in \partial B$. Denote by $u_{l}$ the neighbor of $v$ in the convex path $\partial B$. Since $u_{l}$ and $u_{j}$ are not consecutive if $G$ is a graph of type $(4,4)$ or $(6,3)$, and $u_{l}$ and $u_{j}$ are not consecutive or 2-consecutive if $G$ is a graph of type $(3,6)$, from the first part of this proof we obtain that $v \in I\left(u_{l}, y\right)$.

If the projection of $y$ on $Z(A, B)$ does not intersect the subpath of $\partial B$ starting at $u_{l}$, passing via $z$, and ending at a vertex of $\partial G$, then $v \in I(z, y) \subset I(x, y)$, and we are done. On the other hand, if the whole projection $\operatorname{Pr}(y, Z(A, B))$ is contained in this subpath of $\partial B$, then necessarily $u_{l} \in I(v, y)$. Since $u_{j} \in I(v, y)$, from Lemma 4.9 we conclude that $u_{l}$ and $u_{j}$ are consecutive, i.e., $u_{i}$ and $u_{j}$ must be 2 -consecutive. $\operatorname{So} \operatorname{Pr}(y, Z(A, B))$ must have vertices of $\partial B$ on both sides of $u_{l}$. This is impossible if $G$ is a graph of type $(4,4)$ : by Lemma $4.10, \operatorname{Pr}(y, Z(A, B))$ will consist of two adjacent vertices $v$ and $u_{l}$, however $v \in I\left(u_{l}, y\right)$ as noticed above. If $G$ is a $(3,6)$-graph, then Lemma 4.11 implies that both $v$ and $u_{l}$ belong to $\operatorname{Pr}(y, Z(A, B))$, which is impossible because $v \in I\left(u_{l}, y\right)$ by what has been shown above. This contradiction completes the proof.

\subsection{Partition into cones}

Let $v$ be a median vertex of $G$ (which we assume to be an inner vertex) and let $N(v)=$ $\left\{u_{0}, \ldots, u_{k-1}\right\}$ be the set of neighbors of $v$ ordered counterclockwise around $v$. Every edge $v u_{i}$ is crossed by two alternating cuts $\left\{A_{i}^{\prime}, B_{i}^{\prime}\right\}$ and $\left\{A_{i}^{\prime \prime}, B_{i}^{\prime \prime}\right\}$. Recall that we have chosen an orientation of these cuts such that $v$ is on the left border. Moreover, we suppose that $\left\{A_{i}^{\prime}, B_{i}^{\prime}\right\}$ is the cut for which the last turn before $u_{i} v$ (if it exists) is to the right and thus the next turn after $u_{i} v$ is to the left. For each neighbor $u_{i}$ of $v$, define the cone with apex $u_{i}$ as $C_{v}\left(u_{i}\right):=B_{i}^{\prime} \cap A_{i+1(\bmod k)}^{\prime}$. Let $\Gamma_{i}$ be the closed walk which starts at $u_{i}$ follows $\partial B_{i}^{\prime}$ backward (with respect to the orientation of the cut $\left\{A_{i}^{\prime}, B_{i}^{\prime}\right\}$ ) until a boundary vertex $b_{i} \in \partial G \cap \partial B_{i}^{\prime}$, traverses the boundary $\partial G$ counterclockwise until it meets a vertex $a_{i}$ in $\partial A_{i+1}^{\prime} \cap \partial G$ and then goes back to $u_{i}$ following the subpath of $\partial A_{i+1}^{\prime}$ comprised between $a_{i}$ and $u_{i}$. From the definition of $C_{v}\left(u_{i}\right)$ and Lemma 4.4 it follows that the cone $C_{v}\left(u_{i}\right)$ consists of all vertices of $G$ lying on $\Gamma_{i}$ or inside the region bounded by $\Gamma_{i}$ (see Fig. 4).

Lemma 4.14. $C_{v}\left(u_{i}\right)$ consists of all vertices $x$ such that $I(v, x) \cap N(v)$ equals $\left\{u_{i}\right\}$ or $\left\{u_{i-1}, u_{i}\right\}$. In particular, together with $\{v\}$ the cones $C_{v}\left(u_{i}\right)(i=0, \ldots, k-1)$ constitute $a$ partition of the vertex set of $G$, each set containing at most $n / 2$ vertices.

Proof. We first show that for a vertex $x \in C_{v}\left(u_{i}\right)$ any shortest $(x, v)$-path goes via $u_{i}$ or $u_{i-1}$. Let $y z$ be the first edge on this path such that $y \in C_{v}\left(u_{i}\right)$ and $z \notin C_{v}\left(u_{i}\right)$. Then, either 


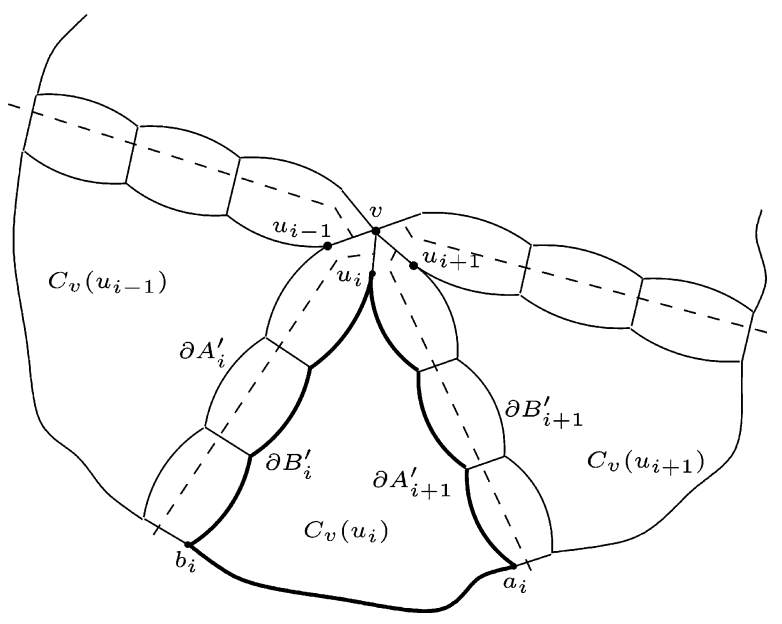

Fig. 4. The cone $C_{v}\left(u_{i}\right)$.

$y \in \partial A_{i+1}^{\prime}$ and $z \in \partial B_{i+1}^{\prime}$ or $y \in \partial B_{i}^{\prime}$ and $z \in \partial A_{i}^{\prime}$. In the first case, since $u_{i}$ is located on the subpath of the convex path $\partial A_{i+1}^{\prime}$ between $y$ and $v$, the unique shortest $(y, v)$-path is a subpath of $\partial A_{i+1}^{\prime}$ which traverses $u_{i}$. In the second case, analogously, either $z=v$ or $u_{i-1}$ is located on the subpath of the convex path $\partial A_{i}^{\prime}$ between $z$ and $v\left(z\right.$ and $u_{i-1}$ may coincide). Therefore, the unique shortest $(z, v)$-path goes via $u_{i-1}$. Hence no shortest $(x, v)$-path goes via a neighbor of $v$ different from $u_{i}$ and $u_{i-1}$. Conversely, let $x$ be a vertex of $G$ such that $I(x, v) \cap N(v)$ equals $\left\{u_{i}\right\}$ or $\left\{u_{i-1}, u_{i}\right\}$. Pick a shortest $(x, v)$-path which goes via $u_{i}$. Pick the first edge $y z$ on this path such that $z \notin C_{v}\left(u_{i}\right)$ and $y \in C_{v}\left(u_{i}\right)$. If $y \in \partial B_{i}^{\prime}$ and $z \in \partial A_{i}^{\prime}$, we obtain a contradiction with the convexity of $\partial A_{i}^{\prime}$ because $z, v \in \partial A_{i}^{\prime}$ while a shortest path joining them leaves $\partial A_{i}^{\prime}$. Otherwise, we have $z \in \partial B_{i+1}^{\prime}$ and $y \in \partial A_{i+1}^{\prime}$. By Lemma 4.4, $z$ belongs to $W\left(u_{i+1}, v\right)$, hence there is a shortest $(z, v)$ path which goes via $u_{i+1}$, in contradiction with the choice of $x$.

Two cones $C_{v}\left(u_{i}\right)$ and $C_{v}\left(u_{j}\right)$ are called $p$-neighboring if $\min \{|i-j|, k-|i-j|\}=p$.

Lemma 4.15. If $x \in C_{v}\left(u_{i}\right)$ and $y \in C_{v}\left(u_{j}\right)$ and the cones $C_{v}\left(u_{i}\right)$ and $C_{v}\left(u_{j}\right)$ are not p-neighboring for $p \leqslant 2$, then $d(x, y)=d(x, v)+d(v, y)$.

Proof. For graphs of type $(4,4)$ (or $(6,3))$ the result directly follows from Lemma 4.13 because $C_{v}\left(u_{i}\right) \subseteq W\left(u_{i}, v\right)$ and $C_{v}\left(u_{j}\right) \subseteq W\left(u_{j}, v\right)$ by Lemma 4.14. The same argument can be applied for $(3,6)$-graphs except for the case when $u_{i}$ and $u_{j}$ are 3 -consecutive. Let $u_{l}$ be as in the proof of Lemma 4.13. Following the same proof, we will obtain the required property $d(x, y)=d(x, v)+d(x, y)$ except the case when $v, u_{l} \in \operatorname{Pr}(y, Z(A, B))$. Then $d(y, v)=d\left(y, u_{l}\right)$. Consider the face $F \notin Z(A, B)$ containing the edge $v u_{l}$ and provided by Lemma 4.8. Then the neighbor $u_{r} \neq u_{l}$ of $v$ in $F$ belongs to the interval $I(v, y)$. Since $u_{j}$ also belongs to $I(v, y)$, from Lemma 4.9 we infer that $u_{r}$ and $u_{j}$ are consecutive. In the partition into cones, $y$ will belong to the cone $C_{v}\left(u_{r}\right)$, which is 2-neighboring with $C_{v}\left(u_{i}\right)$, and not to $C_{v}\left(u_{j}\right)$. 


\section{Distance queries and routing}

In this section, we describe in details the distance and routing labeling schemes in a graph $G$ of type $(4,4)$ and $(3,6)$.

\subsection{Computing the distance between two vertices $x \in A$ and $y \in B$}

In this subsection, given an alternating cut $\{A, B\}$ with the zone $Z(A, B)$, we show how to compute in constant time the distance $d(x, y)$ between two vertices $x \in A$ and $y \in B$. We use the short-hands $P:=\operatorname{Pr}(x, Z(A, B))$ and $Q:=\operatorname{Pr}(y, Z(A, B))$. Recall also that for a vertex $p$ and a subset $S$ of $G$, the distance from $p$ to $S$ is $d(x, S)=\min \{d(x, s): s \in S\}$.

By Lemmas 4.10 and 4.11 , if $G$ is a $(4,4)$ - or a $(3,6)$-graph, then $P$ and $Q$ are paths and the distance $d(x, y)$ can be computed using the formula $d(x, y)=d(x, Z(A, B))+$ $d(P, Q)+d(y, Z(A, B))$. In order to implement this formula in constant time, it suffices to keep at $x$ the distance $d(x, Z(A, B))=d(x, P)$ and two labels allowing to locate in $\partial A$ the end vertices $p^{\prime}, p^{\prime \prime}$ of $P$, and at $y$ the distance $d(y, Z(A, B))=d(y, Q)$ and two labels allowing to locate in $\partial B$ the end vertices $q^{\prime}, q^{\prime \prime}$ of $Q$.

Before describing the procedure distance_paths which reports in constant time the distance between two paths $P \subseteq \partial A$ and $Q \subseteq \partial B$ of the zone $Z(A, B)$, we present an encoding of the vertices of $Z(A, B)$ which allows to compute the distance between any two vertices $p \in \partial A$ and $q \in \partial B$ in $O(1)$ time. Pick an edge $a b \in E(A, B) \cap \partial G$, where $a \in \partial A, b \in \partial B$, and $\partial A$ is the left border line of $Z(A, B)$. Suppose, without loss of generality, that the last turn (if it exists) of $E(A, B)$ before the edge $a b$ is to the right (the other case being analogous). Then for every edge $a^{\prime} b^{\prime} \in Z(A, B)$ with $a^{\prime} \in A$ and $b^{\prime} \in B$ either $d\left(a, a^{\prime}\right)=d\left(b, b^{\prime}\right)$ or $d\left(a, a^{\prime}\right)=d\left(b, b^{\prime}\right)+1$ holds. We say that the edge $a^{\prime} b^{\prime}$ is horizontal in the first case and inclined in the second case. For a vertex $b^{\prime} \in \partial B$ define $\alpha_{1}\left(b^{\prime}\right):=\min \left\{d\left(a^{\prime}, a\right)-d\left(b^{\prime}, b\right): a^{\prime} b^{\prime} \in E(A, B)\right\}$ and $\alpha_{2}\left(b^{\prime}\right):=$ $\max \left\{d\left(a^{\prime}, a\right)-d\left(b^{\prime}, b\right): a^{\prime} b^{\prime} \in E(A, B)\right\}$. If $G$ is of type $(4,4)$ then $b^{\prime}$ has a unique neighbor in $\partial A$ (because $\partial A$ is convex by Lemma 4.3 and $G$ is triangle-free), and thus $\alpha_{1}\left(b^{\prime}\right)=\alpha_{2}\left(b^{\prime}\right) \in\{0,1\}$. On the other hand, if $G$ is of type $(3,6)$ and $b^{\prime}$ belongs to a triangular face of the zone $Z(A, B)$, then we may have $\alpha_{1}\left(b^{\prime}\right)=0$ and $\alpha_{2}\left(b^{\prime}\right)=1$. We say that a vertex $a^{\prime} \in \partial A$ is above vertex $p \in \partial A$ if it belongs to the subpath of $\partial A$ comprised between $p$ and $a$, and below $p$ otherwise (we employ the same terminology for vertex $q \in \partial B$ and the vertices $\left.b^{\prime} \in \partial B\right)$. By convention, $p$ is above and below itself. Let $r(p)$ be the first vertex above $p$ which is incident to an inclined edge of $E(A, B)$ and let $s(p)$ be the first vertex below $p$ which is incident to a horizontal edge of $E(A, B)$ (if such vertices do not exist, then set $r(p):=a$ and let $s(p)$ be the second end vertex of $\partial A)$. Let above $(p)$ and below $(p)$ be the first vertices above and below $p$ which are incident to edges of $E(A, B)$ (notice that above $(p)=p=\operatorname{below}(p)$ if $p$ has a neighbor in $\partial B$ ). Let also Above $(p)$ (with capital A) be the first vertex strictly above $p$ which is incident to an edge of $E(A, B)$ (if $p=a$ we set Above $(p):=p)$. Clearly, unless $p=a$, Above $(p) \neq p$ holds. Analogously define above $(q)$, Above $(q)$ and below $(q)$ for the vertices $q \in \partial B$. We say that a vertex $p \in \partial A$ is above a vertex $q \in \partial B$ if $d(a$, below $(p)) \leqslant d(b$, above $(q))+\alpha_{2}(\operatorname{above}(q))$ (in a similar way, we say $q$ is above $p$ if $d(b, \operatorname{below}(q))+\alpha_{1}(\operatorname{below}(q)) \leqslant d(a$,above $\left.(p))\right)$. 
Using the labels introduced in previous paragraph, we can recognize in $O(1)$ time if two vertices $p^{\prime}, p^{\prime \prime} \in \partial A$ belong to a common face of $Z(A, B)$ : this holds if and only if

$$
\begin{aligned}
& d\left(a, \operatorname{Above}\left(p^{\prime}\right)\right)=d\left(a, \operatorname{Above}\left(p^{\prime \prime}\right)\right) \text { or } \\
& d\left(a, \operatorname{below}\left(p^{\prime}\right)\right)=d\left(a, p^{\prime}\right)=d\left(a, \operatorname{Above}\left(p^{\prime \prime}\right)\right)
\end{aligned}
$$

(a similar test can be applied to the vertices $q^{\prime}, q^{\prime \prime} \in \partial B$ ). Analogously, one can test in $O$ (1) time if two vertices $p \in \partial A$ and $q \in \partial B$ belong to a common face of $Z(A, B)$. This happens if and only if at least one of the equalities

$$
\begin{aligned}
& (p=a \text { and } q=b) \quad \text { or } d(a, \text { Above }(p))=d(b, \text { Above }(q))+\alpha_{2}(\operatorname{Above}(q)) \quad \text { or } \\
& d(a, \operatorname{below}(p))=d(a, p)=d(b, \operatorname{Above}(q))+\alpha_{2}(\operatorname{Above}(q)) \quad \text { or } \\
& d(b, \operatorname{below}(q))=d(b, q)=d(a, \operatorname{Above}(p))-\alpha_{2}(\operatorname{above}(q)) \quad \text { or } \\
& d(a, \operatorname{Above}(p))=d(a, p)-1=d(b, q)=d(b, \operatorname{below}(q))
\end{aligned}
$$

holds. We call this the common_face_test (its formal description is given below).

If for two vertices $p \in \partial A$ and $q \in \partial B$, one is above another one, then a simple case analysis shows that $d(p, q)$ can be computed via the formula (see Fig. 5(a))

$$
d(p, q)=|d(p, a)-d(q, b)|+\varepsilon,
$$

where

$$
\varepsilon= \begin{cases}0 & \text { if } d(q, b)<d(r(p), a), \\ 1 & \text { if } d(r(p), a) \leqslant d(q, b)<d(p, a) \text { or } d(s(p), a) \leqslant d(q, b), \\ 2 & \text { if } d(p, a) \leqslant d(q, b)<d(s(p), a) .\end{cases}
$$

Otherwise, one can see that $p$ and $q$ belong to a common face of $Z(A, B)$ and that $d(p, q)$ is the minimum of

$$
\begin{aligned}
& d(a, p)-d(a, \text { above }(p))+1+d(b, q)-d(b, \text { above }(q)) \quad \text { and } \\
& d(a, \operatorname{below}(p))-d(a, p)+1+d(b, \operatorname{below}(q))-d(b, q) .
\end{aligned}
$$

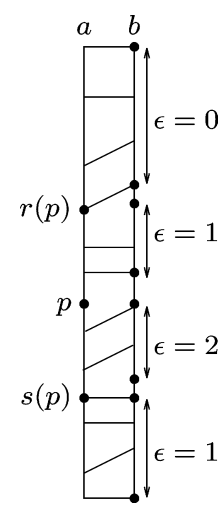

(a) To formula (1).

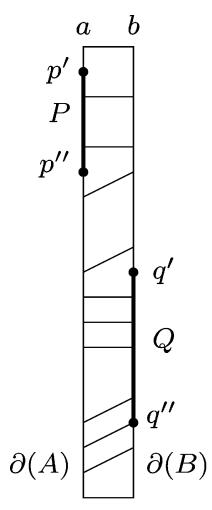

(i)

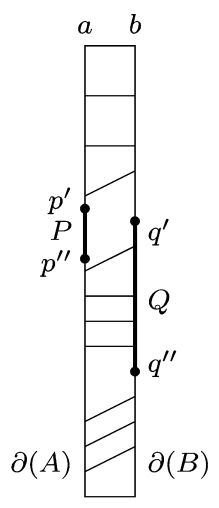

(ii)

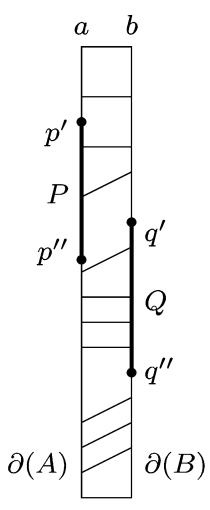

(iii)

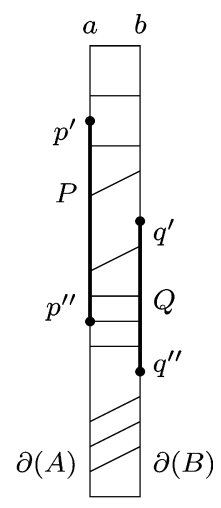

(iv)

(b) Cases (i)-(iv).

Fig. 5. 
Therefore, we can retrieve $d(p, q)$ in $O(1)$ time from the label

$$
\begin{aligned}
B_{p}:= & \stackrel{1}{1} \underset{2}{ } \stackrel{2}{d}(p, a), d(\operatorname{above}(p), a), d(\operatorname{below}(p), a), d(s(p), a), d(r(p), a), \\
& d(\operatorname{Above}(p), a))
\end{aligned}
$$

of $p \in \partial A$ and the label

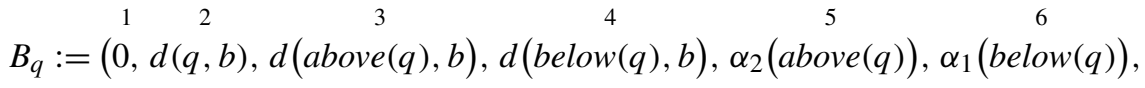

$$
\begin{aligned}
& \begin{array}{c}
7 \\
d(\operatorname{Above}(q), b) \\
\left.\alpha_{2}(\operatorname{Above}(q))\right)
\end{array}
\end{aligned}
$$

of $q \in \partial B$ (see function distance_vertices $\left(B_{p}, B_{q}\right)$ below). The first entry in $B_{p}$ (and in $B_{q}$ ) is a bit that indicates that the last turn (if it exists) of $E(A, B)$, before the edge $a b$, is to the right. If the last turn is to the left, we need to interchange the roles of $p$ and $q$ in the consideration.

Notice also that $d\left(p^{\prime}, p^{\prime \prime}\right)=\left|d\left(a, p^{\prime}\right)-d\left(a, p^{\prime \prime}\right)\right|$ for any two vertices $p^{\prime}, p^{\prime \prime} \in \partial A$ and $d\left(q^{\prime}, q^{\prime \prime}\right)=\left|d\left(b, q^{\prime}\right)-d\left(b, q^{\prime \prime}\right)\right|$ for any two vertices $q^{\prime}, q^{\prime \prime} \in \partial B$.

function distance_vertices $\left(B_{p}, B_{q}\right)$

if $B_{p}(1)=0$ then $/ *$ rename inputs $* /$ set $C:=B_{p}, B_{p}:=B_{q}, B_{q}:=C$

if $B_{p}(4) \leqslant B_{q}(3)+B_{q}(5)$ or $B_{q}(4)+B_{q}(6) \leqslant B_{p}(3)$ then

if $B_{q}(2)<B_{p}(6)$ then set $\varepsilon:=0$

if $B_{p}(6) \leqslant B_{q}(2)<B_{p}(2)$ or $B_{p}(5) \leqslant B_{q}(2)$ then set $\varepsilon:=1$

if $B_{p}(2) \leqslant B_{q}(2)<B_{p}(5)$ then set $\varepsilon:=2$

return $\left|B_{p}(2)-B_{q}(2)\right|+\varepsilon$.

else

$$
\begin{array}{r}
\text { return } \min \left\{B_{p}(2)-B_{p}(3)+1+B_{q}(2)-B_{q}(3),\right. \\
\left.B_{p}(4)-B_{p}(2)+1+B_{q}(4)-B_{q}(2)\right\}
\end{array}
$$

function common_face_test $\left(B_{p}, B_{q}\right.$, flag)

do case

case flag $=0 / *$ i.e., $p$ and $q$ are on the same side of the zone */

if $B_{p}(7)=B_{q}(7)$ or $B_{p}(4)=B_{p}(2)=B_{q}(7)$ then return true

else return false

case flag $=1 / *$ i.e., $p$ and $q$ are on different sides of the zone $* /$

if $B_{p}(1)=0$ then $/ *$ rename inputs $* /$ set $C:=B_{p}, B_{p}:=B_{q}, B_{q}:=C$

if $B_{p}(2)=B_{q}(2)=0$ or $B_{p}(7)=B_{q}(7)+B_{q}(8)$ or

$B_{p}(4)=B_{p}(2)=B_{q}(7)+B_{q}(8)$ or $B_{q}(4)=B_{q}(2)=B_{p}(7)+B_{q}(5)$ or

$B_{p}(7)=B_{p}(2)-1=B_{q}(2)=B_{q}(4)$ then return true

end case.

else return false

Now we will adjust this labeling scheme in order to compute the distance $d(P, Q):=$ $\min \{d(p, q): p \in P, q \in Q\}$ between two paths $P \subseteq \partial A$ and $Q \subseteq \partial B$. Let $p^{\prime}, p^{\prime \prime}$ be the 
end vertices of $P$ and let $q^{\prime}, q^{\prime \prime}$ be the end vertices of $Q$, such that $d\left(p^{\prime}, a\right) \leqslant d\left(p^{\prime \prime}, a\right)$ and $d\left(q^{\prime}, b\right) \leqslant d\left(q^{\prime \prime}, b\right)$. We distinguish between four complementary cases depending of the reciprocal location of $P$ and $Q$; see Fig. 5(b) for an illustration:

(i) one path is above another;

(ii) one path is contained in a face of $Z(A, B)$ and the second path intersects this face;

(iii) there is a unique face $F$ of $Z(A, B)$ intersecting both paths $P, Q$ and neither of these paths is contained in $F$;

(iv) several faces of $Z(A, B)$ intersect both paths $P$ and $Q$.

We say that path $P$ is above $Q$ if $p^{\prime \prime}$ is above $q^{\prime}$. In this case, $d(P, Q)=d\left(p^{\prime \prime}, q^{\prime}\right)$. Analogously, $Q$ is above $P$ if $q^{\prime \prime}$ is above $p^{\prime}$. Then $d(P, Q)=d\left(p^{\prime}, q^{\prime \prime}\right)$. This settles case (i), therefore further we may suppose that neither $P$ is above $Q$ nor $Q$ is above $P$.

Applying the common_face_test, we can check in constant time if any pair of the vertices $p^{\prime}, p^{\prime \prime}, q^{\prime}, q^{\prime \prime}$ belong to a common face of $Z(A, B)$. Suppose this test returned that $p^{\prime}$ and $p^{\prime \prime}$ belong to a common face, say $F$. If $q^{\prime}$ and $q^{\prime \prime}$ also belong to a common face (which cannot be other than $F$ ), then

$$
d(P, Q)=\min \left\{d\left(p^{\prime}, q^{\prime}\right), d\left(p^{\prime \prime}, q^{\prime \prime}\right)\right\} .
$$

If $q^{\prime} \in F$ and $q^{\prime \prime} \notin F$ (employing the common_face_test for $p^{\prime}, q^{\prime}$ and $p^{\prime}, q^{\prime \prime}$ ), then

$$
d(P, Q)=\min \left\{d\left(p^{\prime}, q^{\prime}\right), d\left(p^{\prime \prime}, \operatorname{below}\left(p^{\prime \prime}\right)\right)+1\right\},
$$

because the neighbor of below $\left(p^{\prime \prime}\right)$ in $\partial B$ will be a vertex of $Q$ (a similar formula holds if $q^{\prime \prime} \in F$ and $q^{\prime}$ does not belong to $F$ ). Finally, if both vertices $q^{\prime}$ and $q^{\prime \prime}$ are outside $F$, then

$$
d(P, Q)=\min \left\{d\left(p^{\prime}, \text { above }\left(p^{\prime}\right)\right)+1, d\left(p^{\prime \prime}, \operatorname{below}\left(p^{\prime \prime}\right)\right)+1\right\} .
$$

This completes the analysis of case (ii), so further we may assume that neither of the paths $P$ and $Q$ is entirely contained in a face of $Z(A, B)$.

The case (iii) arises if and only if only one of the pair of vertices $p^{\prime}, q^{\prime \prime}$ and $p^{\prime \prime}, q^{\prime}$ belongs to a common face of $Z(A, B)$ and this again can be detected by the common_face_test. In the first case,

$$
d(P, Q)=\min \left\{d\left(p^{\prime}, \text { above }\left(p^{\prime}\right)\right)+1, d\left(q^{\prime \prime}, \operatorname{below}\left(q^{\prime \prime}\right)\right)+1\right\} .
$$

In the second case,

$$
d(P, Q)=\min \left\{d\left(q^{\prime}, \text { above }\left(q^{\prime}\right)\right)+1, d\left(p^{\prime \prime}, \operatorname{below}\left(p^{\prime \prime}\right)\right)+1\right\} .
$$

Finally, if no condition of cases (i)-(iii) is satisfied, then several faces of $Z(A, B)$ intersect both paths $P$ and $Q$. Then obviously there is an edge of $E(A, B)$ with one end in $P$ and another end in $Q$, yielding $d(P, Q)=1$.

Summarizing, we conclude that from the labels $B_{p^{\prime}}, B_{p^{\prime \prime}}, B_{q^{\prime}}$, and $B_{q^{\prime \prime}}$ of the end vertices of the paths $P \subseteq \partial A$ and $Q \subseteq \partial B$ we can compute the distance $d(P, Q)$ in $O(1)$ time. We call this subroutine distance_paths. 
function distance_paths $\left(B_{p^{\prime}}, B_{p^{\prime \prime}}, B_{q^{\prime}}, B_{q^{\prime \prime}}\right)$

if $B_{p^{\prime}}(1)=0$ then $/ *$ rename inputs $* /$ set $C:=B_{p^{\prime}}, B_{p^{\prime}}:=B_{q^{\prime}}, B_{q^{\prime}}:=C$

and $C:=B_{p^{\prime \prime}}, B_{p^{\prime \prime}}:=B_{q^{\prime \prime}}, B_{q^{\prime \prime}}:=C$

if $B_{p^{\prime \prime}}(4) \leqslant B_{q^{\prime}}(3)+B_{q^{\prime}}(5)$ then return distance_vertices $\left(B_{p^{\prime \prime}}, B_{q^{\prime}}\right)$

if $B_{q^{\prime \prime}}(4)+B_{q^{\prime \prime}}(6) \leqslant B_{p^{\prime}}(3)$ then return distance_vertices $\left(B_{p^{\prime}}, B_{q^{\prime \prime}}\right)$

if common_face_test $\left(B_{p^{\prime}}, B_{p^{\prime \prime}}, 0\right)$ then

if common_face_test $\left(B_{q^{\prime}}, B_{q^{\prime \prime}}, 0\right)$ then

return $\min \left\{\right.$ distance_vertices $\left(B_{p^{\prime}}, B_{q^{\prime}}\right)$, distance_vertices $\left.\left(B_{p^{\prime \prime}}, B_{q^{\prime \prime}}\right)\right\}$

if common_face_test $\left(B_{p^{\prime}}, B_{q^{\prime}}, 1\right)$ and $\operatorname{not}\left(\operatorname{common} \_f a c e \_t e s t\left(B_{p^{\prime}}, B_{q^{\prime \prime}}, 1\right)\right)$ then

return $\min \left\{\right.$ distance_vertices $\left.\left(B_{p^{\prime}}, B_{q^{\prime}}\right), B_{p^{\prime \prime}}(4)-B_{p^{\prime \prime}}(2)+1\right\}$

if $\operatorname{not}\left(\right.$ common_face_test $\left.\left(B_{p^{\prime}}, B_{q^{\prime}}, 1\right)\right)$ and common_face_test $\left(B_{p^{\prime}}, B_{q^{\prime \prime}}, 1\right)$ then

return min $\left\{\right.$ distance_vertices $\left(B_{p^{\prime \prime}}, B_{q^{\prime \prime}}\right), B_{p^{\prime}}(2)-B_{p^{\prime}}(3)+1$ \}

else return $\min \left\{B_{p^{\prime}}(2)-B_{p^{\prime}}(3)+1, B_{p^{\prime \prime}}(4)-B_{p^{\prime \prime}}(2)+1\right\}$

if common_face_test $\left(B_{q^{\prime}}, B_{q^{\prime \prime}}, 0\right)$ then

if common_face_test $\left(B_{p^{\prime}}, B_{q^{\prime}}, 1\right)$ then

return min $\left\{\right.$ distance_vertices $\left(B_{p^{\prime}}, B_{q^{\prime}}\right), B_{q^{\prime \prime}}(4)-B_{q^{\prime \prime}}(2)+1$ \}

if common_face_test $\left(B_{p^{\prime \prime}}, B_{q^{\prime}}, 1\right)$ then

return $\min \left\{\right.$ distance_vertices $\left(B_{p^{\prime \prime}}, B_{q^{\prime \prime}}\right), B_{q^{\prime}}(2)-B_{q^{\prime}}(3)+1$ \}

else return $\min \left\{B_{q^{\prime}}(2)-B_{q^{\prime}}(3)+1, B_{q^{\prime \prime}}(4)-B_{q^{\prime \prime}}(2)+1\right\}$

if common_face_test $\left(B_{p^{\prime}}, B_{q^{\prime \prime}}, 1\right)$ then

return $\min \left\{B_{p^{\prime}}(2)-B_{p^{\prime}}(3)+1, B_{q^{\prime \prime}}(4)-B_{q^{\prime \prime}}(2)+1\right\}$

if common_face_test $\left(B_{p^{\prime \prime}}, B_{q^{\prime}}, 1\right)$ then

return $\min \left\{B_{p^{\prime \prime}}(4)-B_{p^{\prime \prime}}(2)+1, B_{q^{\prime}}(2)-B_{q^{\prime}}(3)+1\right\}$

else return 1 .

Summarizing all discussions of this subsection, we can retrieve $d(x, y)$ in $O(1)$ time from the label

$$
D_{x}:=\left(d\left(x, p^{\prime}\right), d(x, Z(A, B)), d\left(x, p^{\prime \prime}\right), B_{p^{\prime}}, B_{p^{\prime \prime}}\right)
$$

of $x \in A$ and the label

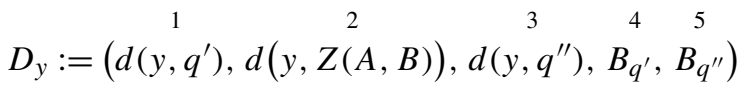

of $y \in B$ using function distance_graphs $\left.\left(D_{x}, D_{y}\right)\right)$ given below:

function distance_graphs $\left(D_{x}, D_{y}\right)$

return $D_{x}(2)+$ distance_paths $\left(D_{x}(4), D_{x}(5), D_{y}(4), D_{y}(5)\right)+D_{y}(2)$

\subsection{Distance decoder}

Here we explain how, using the decomposition tree $T(G)$, one can find the distance between any two vertices of $G$. First, we will describe the labels of vertices of $G$.

Let $v$ be a median vertex of $G$ (which we assume to be an inner vertex), and let $u_{0}, \ldots, u_{k-1}$ be its neighbors in counterclockwise order around $v$. Recall that the cones $C_{v}\left(u_{i}\right), i \in\{0, \ldots, k-1\}$ of $G$ were defined as follows: $C_{v}\left(u_{i}\right)=B_{i}^{\prime} \cap A_{i+1(\bmod k)}^{\prime}$. Each 
vertex $y \in V \backslash C_{v}\left(u_{i}\right)$ is separated from a vertex $x \in C_{v}\left(u_{i}\right)$ by zone $Z\left(A_{i}^{\prime}, B_{i}^{\prime}\right)$ or by zone $Z\left(A_{i+1}^{\prime}, B_{i+1}^{\prime}\right)$. From previous results we know that, if two vertices $x$ and $y$ lie in two 1 -neighboring or 2-neighboring cones, then $d(x, y)$ is realized via their projections on the zone separating these cones, and if $x$ and $y$ belong to $p$-neighboring cones with $p>2$, then $d(x, y)$ is realized via $v$. For any vertex $x \in C_{v}\left(u_{i}\right)$ and index $j=i, i+1, i+2(\bmod k)$, let $D_{x}^{j}$ be the distance label of $x$ with respect to the cut $\left\{A_{j}^{\prime}, B_{j}^{\prime}\right\}$ ( $D_{x}$ was defined in the previous subsection with respect to an arbitrary cut $\{A, B\})$. Let also $G_{i}$ be a subgraph of $G$ induced by $C_{v}\left(u_{i}\right)(i \in\{0, \ldots, k-1\})$.

Assume that a decomposition tree $T(G)$ of $G$ and its NCA-depth labeling scheme are given. For a vertex $x$ of $G$, let $S(x)$ be the deepest node of $T(G)$ containing $x$ and $A_{x}$ be the label of $S(x)$ in the NCA-depth labeling scheme. Let also $S_{0}, S_{1}, \ldots, S_{h}$ be the nodes of the path of $T(G)$ from the root $(G, v)$ (which is $\left.S_{0}\right)$ to the node $S(x)=S_{h}$.

In the distance labeling scheme for $(4,4)$ - and $(3,6)$-graphs, the label $L(x)$ will be the concatenation of $A_{x}$, and $h+1$ tuples $\tau_{0}^{x}, \tau_{1}^{x}, \ldots, \tau_{h}^{x}$ where $\tau_{q}^{x}(q \in\{0, \ldots, h\})$ is defined as follows. Let $S_{q}$ be a node $\left(G_{q}, v_{q}\right)$ of $T(G)$. Assume that $x$ belongs to a cone $C_{v_{q}}\left(u_{i}\right)$ of $G_{q}$ for some $i \in\left\{0, \ldots, \delta_{G_{q}}\left(v_{q}\right)-1\right\}$. Then,

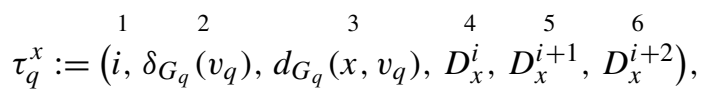

where zones and projections are considered in graph $G_{q}$. If $x=v_{q}$, we set $\tau_{q}^{v_{q}}:=$ $\left(\delta_{G_{q}}\left(v_{q}\right), \delta_{G_{q}}\left(v_{q}\right), 0,0,0,0\right)$.

Since the depth of $T(G)$ is $O(\log n), L(x)$ is of length $O\left(\log ^{2} n\right)$ bits for any $x \in V$. Note that computation of those tuples can be incorporated into the algorithm of building $T(G)$, leading to an $O\left(n^{2} \log n\right)$ time computation of all labels $L(x), x \in V$ (for a graph $G_{q}$, the paths $\operatorname{Pr}\left(x, Z\left(A_{j}^{\prime}, B_{j}^{\prime}\right)\right)(j=i, i+1, i+2)$ and corresponding distances can be computed by running Bread-First-Searches from $v_{q}$ and $\left.Z\left(A_{j}^{\prime}, B_{j}^{\prime}\right)(j=i, i+1, i+2)\right)$.

Algorithm DISTANCE_DECODER: Distance decoder for $(4,4)$ and $(3,6)$-graphs.

Input: two labels $L(x)=A_{x} \circ \tau_{0}^{x} \circ \tau_{1}^{x} \circ \cdots \circ \tau_{h}^{x}$ and $L(y)=A_{y} \circ \tau_{0}^{y} \circ \tau_{1}^{y} \circ \cdots \circ \tau_{q}^{y}$.

Output: $d(x, y)$, the distance between $x$ and $y$ in $G$.

Method:

use $A_{x}$ and $A_{y}$ to find the depth $l$ in $T(G)$ of

the nearest common ancestor of $S(x)$ and $S(y)$;

extract from $L(x)$ and $L(y)$ the tuples $\tau_{l}^{x}$ and $\tau_{l}^{y}$;

if $\tau_{l}^{x}(1)=\tau_{l}^{x}(2)$ then output $\tau_{l}^{y}(3)$ and stop; $/ * x=v_{q} * /$

if $\tau_{l}^{y}(1)=\tau_{l}^{y}(2)$ then output $\tau_{l}^{x}(3)$ and stop; $/ * \quad y=v_{q} * /$

$/^{*}$ if the cones are 1-neighboring $* /$

if $\left(\tau_{l}^{x}(1)=\tau_{l}^{y}(1)-1\right.$ or $\tau_{l}^{y}(1)=0$ and $\left.\tau_{l}^{x}(1)=\tau_{l}^{x}(2)-1\right)$ then output distance_graphs $\left(\tau_{l}^{x}(5), \tau_{l}^{y}(4)\right)$ and stop;

if $\left(\tau_{l}^{y}(1)=\tau_{l}^{x}(1)-1\right.$ or $\tau_{l}^{x}(1)=0$ and $\left.\tau_{l}^{y}(1)=\tau_{l}^{x}(2)-1\right)$ then output distance_graphs $\left(\tau_{l}^{y}(5), \tau_{l}^{x}(4)\right)$ and stop;

/* if the cones are 2-neighboring */

if $\left(\tau_{l}^{x}(1)=\tau_{l}^{y}(1)-2\right.$ or $\tau_{l}^{y}(1)=0$ and $\tau_{l}^{x}(1)=\tau_{l}^{x}(2)-2$ or 


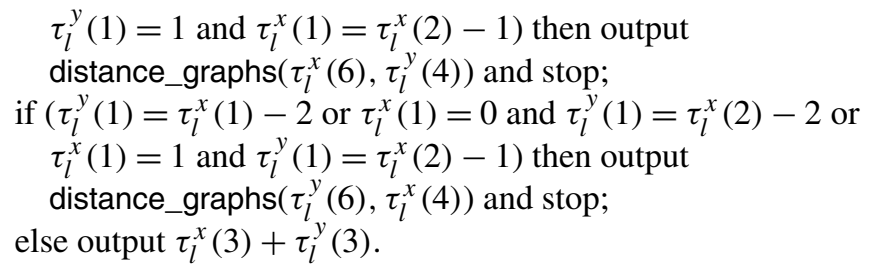

\subsection{Routing from $x \in A$ to $y \in B$}

From Lemma 4.12 we know that any vertex $x \in A$ contains one or two neighbors $v_{x}$ and $u_{x}$ such that $I(x, y) \cap\left\{v_{x}, u_{x}\right\} \neq \emptyset$ for any vertex $y \in B$. Thus the message from $x$ should be forwarded to that of these neighbors which is closer to $y$. If $x \in A \backslash \partial A$, then $u_{x}, v_{x} \in A$ and this decision can be taken in $O(1)$ time by decoding the distances $d\left(y, v_{x}\right)$ and $d\left(y, u_{x}\right)$. Define $h e l p\left(v_{x}\right)$ to be equal to 1 if $x$ and $v_{x}$ are separated by the cut $\{A, B\}$ and 0 otherwise $\left(h e l p\left(u_{x}\right)\right.$, help $\left(v_{y}\right)$, help $\left(u_{y}\right)$ are defined analogously). Then, in this case we can make a routing decision in $O(1)$ time from the label

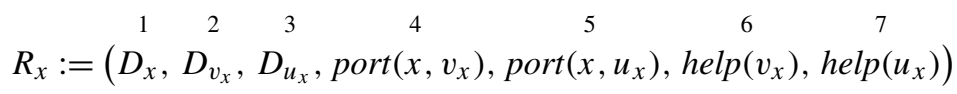

of $x$ and the label $D_{y}$ of $y \in B$ (vice versa, to route from $y \in B \backslash \partial B$ to $x \in A$ we need the label

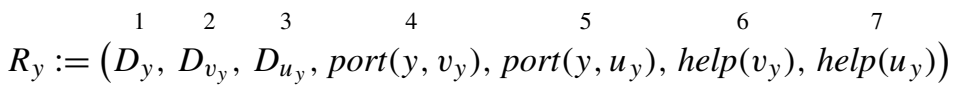

of $y$ and the label $D_{x}$ of $x$ ).

We assert that the same labels $R_{x}$ and $R_{y}$ suffice for the routing decision in case $x \in \partial A$. By second assertion of Lemma 4.12, the neighbors $u_{x}$ and $v_{x}$ of $x$ either both are vertices of $\partial A$, or one of them belong to $\partial A$ and another to $\partial B$, or both are vertices of $\partial B$. In the first case, both distances $d\left(u_{x}, y\right)$ and $d\left(v_{x}, y\right)$ can be decoded as before. In the second case only the distance from $y$ to the vertex of $\partial A$ can be decoded using $R_{x}$ and $R_{y}$, say $d\left(u_{x}, y\right)$. If $d(x, y)=d\left(u_{x}, y\right)+1$, then the message is forwarded to $u_{x}$, otherwise it is sent to $v_{x}$. Finally, if $u_{x}, v_{x} \in \partial B$, then $G$ is a $(3,6)$-graph and the routing decision can be taken by employing the items $d\left(b, q^{\prime}\right), d\left(b, q^{\prime \prime}\right)$ of $D_{y}$ (here $q^{\prime}$ and $q^{\prime \prime}$ are the end vertices of $\operatorname{Pr}(y, Z(A, B))$. Namely, if $u_{x}$ is above $v_{x}$, then the message is forwarded to $u_{x}$ if $d\left(b, q^{\prime \prime}\right) \leqslant d\left(b, u_{x}\right)$ and to $v_{x}$ otherwise.

function routing_decision $\left(R_{x}, R_{y}\right)$

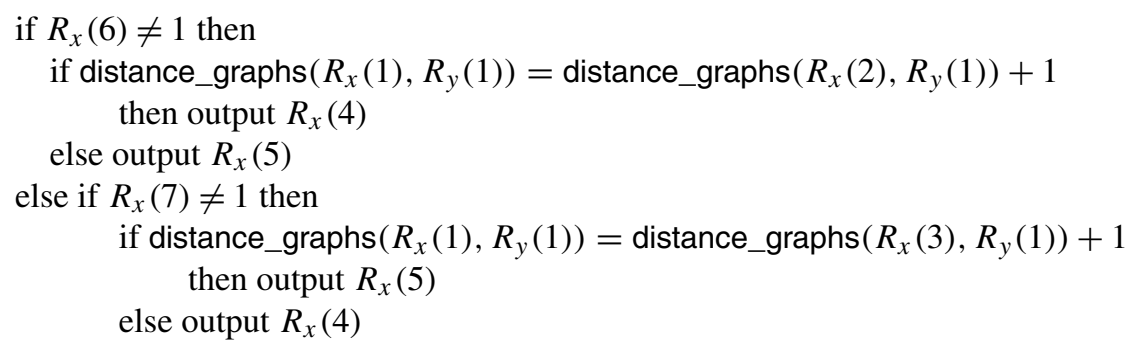


else extract $B_{q^{\prime \prime}}$ from $R_{y}(1)$

extract $B_{u_{x}}$ from $R_{x}(3)$

if $B_{q^{\prime \prime}}(2) \leqslant B_{u_{x}}(2)$ then output $R_{x}(5)$ else output $R_{x}(4)$

\subsection{Routing decision}

Here we explain how, using the decomposition tree $T(G)$, one can rout between any two vertices of $G$. The method is very similar to the one we used for distance decoding.

Let again $v$ be a median vertex of $G$ and $u_{0}, \ldots, u_{k-1}$ be its neighbors in counterclockwise order around $v$. For any vertex $x \in C_{v}\left(u_{i}\right)$ and index $j=i, i+1, i+2(\bmod k)$, denote by $R_{x}^{j}$ the routing label of $x$ with respect to the cut $\left\{A_{j}^{\prime}, B_{j}^{\prime}\right\}$ ( $R_{x}$ was defined in the previous subsection with respect to an arbitrary cut $\{A, B\}$ ). Let $S(x)$ be the deepest node of the decomposition tree $T(G)$ of $G$ containing $x$ and $A_{x}$ be the label of $S(x)$ in the NCA-depth labeling scheme of $T(G)$. Let also $S_{0}, S_{1}, \ldots, S_{h}$ be the nodes of the path of $T(G)$ from the root $(G, v)$ (which is $S_{0}$ ) to the node $S(x)=S_{h}$. Denote as before by $G_{i}$ a subgraph of $G$ induced by $C_{v}\left(u_{i}\right)(i \in\{0, \ldots, k-1\})$.

In the routing labeling scheme for $(4,4)$ - and $(3,6)$-graphs, the label $L(x)$ will be the concatenation of $A_{x}$, and $h+1$ tuples $\mu_{0}^{x}, \mu_{1}^{x}, \ldots, \mu_{h}^{x}$ where $\mu_{q}^{x}(q \in\{0, \ldots, h\})$ is defined as follows. Let $S_{q}$ be a node $\left(G_{q}, v_{q}\right)$ of $T(G)$. Assume that $x$ belongs to a cone $C_{v_{q}}\left(u_{i}\right)$ of $G_{q}$ for some $i \in\left\{0, \ldots, \delta_{G_{q}}\left(v_{q}\right)-1\right\}$. Then,

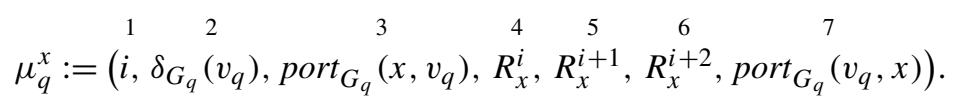

If $x=v_{q}$, we set $\mu_{q}^{v_{q}}:=\left(\delta_{G_{q}}\left(v_{q}\right), \delta_{G_{q}}\left(v_{q}\right), 0,0,0,0,0\right)$.

Clearly, again $L(x)$ is of length $O\left(\log ^{2} n\right)$ bits for any $x \in V$ and computation of those tuples can be incorporated into the algorithm for building $T(G)$, leading to an $O\left(n^{2} \log n\right)$ time computation of all labels $L(x), x \in V$ (for a vertex $x$ of a graph $G_{q}$, the special neighbors $v_{x}^{j}$ and $u_{x}^{j}$ can be computed by running Bread-First-Searches from $Z\left(A_{j}^{\prime}, B_{j}^{\prime}\right)$ $(j=i, i+1, i+2))$.

Algorithm ROUTING_DECISION: Routing decision for $(4,4)$ and $(3,6)$-graphs.

Input: two labels $L(x)=A_{x} \circ \mu_{0}^{x} \circ \mu_{1}^{x} \circ \cdots \circ \mu_{h}^{x}$ and $L(y)=A_{y} \circ \mu_{0}^{y} \circ \mu_{1}^{y} \circ \cdots \circ \mu_{q}^{y}$.

Output: $\operatorname{port}_{G}(x, y)$, the output port number of the first edge on a shortest path from $x$ to $y$ in $G$.

\section{Method:}

use $A_{x}$ and $A_{y}$ to find the depth $l$ in $T(G)$ of

the nearest common ancestor of $S(x)$ and $S(y)$;

extract from $L(x)$ and $L(y)$ the tuples $\mu_{l}^{x}$ and $\mu_{l}^{y}$;

if $\mu_{l}^{x}(1)=\mu_{l}^{x}(2)$ then output $\mu_{l}^{y}(7)$ and stop; /* $x=v_{q} * /$

if $\mu_{l}^{y}(1)=\mu_{l}^{y}(2)$ then output $\mu_{l}^{x}$ (3) and stop; /* $y=v_{q} * /$

$/ * \quad$ if the cones are 1-neighboring $* /$

if $\left(\mu_{l}^{x}(1)=\mu_{l}^{y}(1)-1\right.$ or $\mu_{l}^{y}(1)=0$ and $\left.\mu_{l}^{x}(1)=\mu_{l}^{x}(2)-1\right)$ then output routing_decision $\left(\mu_{l}^{x}(5), \mu_{l}^{y}(4)\right)$ and stop; 


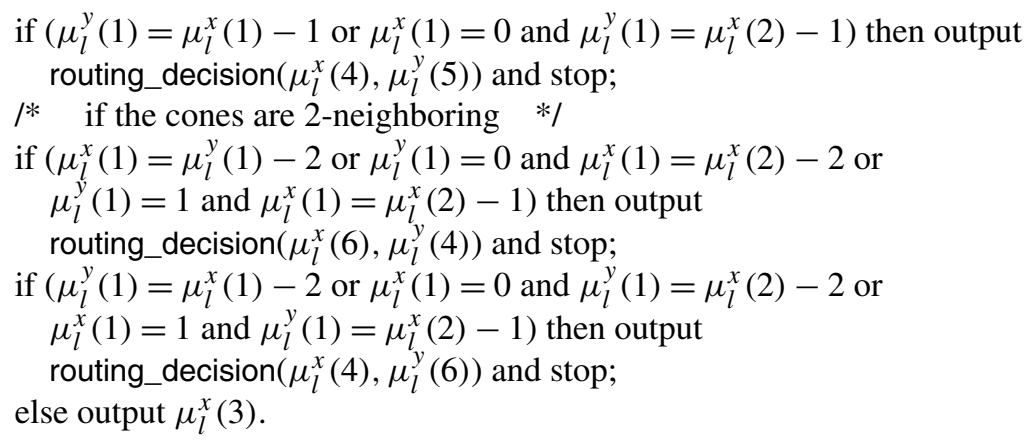

\section{Acknowledgments}

The authors are grateful to two anonymous referees for many helpful suggestions.

\section{References}

[1] E.M. Bakker, J. van Leeuwen, R.B. Tan, Linear interval routing, Algorithms Rev. 2 (1991) 45-61.

[2] H.-J. Bandelt, V. Chepoi, Decomposition and $l_{1}$-embedding of weakly median graphs, European J. Combin. 21 (2000) 701-714.

[3] H.-J. Bandelt, V. Chepoi, Unpublished manuscript.

[4] O. Baues, N. Peyerimhoff, Curvature and geometry of tessellating plane graphs, Discrete Comput. Geom. 25 (2001) 141-159.

[5] M.A. Breuer, Coding the vertices of a graph, IEEE Trans. Inform. Theory IT-12 (1966) 148-153.

[6] M.A. Breuer, J. Folkman, An unexpected result on coding the vertices of a graph, J. Math. Anal. Appl. 20 (1967) 583-600.

[7] V. Chepoi, M. Deza, V. Grishukhin, Clin d'oeil on $L_{1}$-embeddable planar graphs, Discrete Appl. Math. 80 (1997) 3-19.

[8] V. Chepoi, F. Dragan, Y. Vaxès, Distance and routing labeling schemes for non-positively curved plane graphs, Technical report 13-2003, Laboratoire d'Informatique Fondamentale de Marseille, http://www.lim.univ-mrs.fr/Rapports/13-2003-Chepoi-Dragan-Vaxes.pdf.

[9] V. Chepoi, A. Rollin, Interval routing in some planar networks, Theoret. Comput. Sci. 290 (2003) 15031540 .

[10] A.W.M. Dress, R. Scharlau, Gated sets in metric spaces, Aequationes Math. 34 (1987) 112-120.

[11] P. Fraigniaud, C. Gavoille, Routing in trees, in: Proc. 28th Int. Colloquium on Automata, Languages and Programming, ICALP 2001, in: Lecture Notes in Comput. Sci., vol. 2076, 2001, pp. 757-772.

[12] P. Fraigniaud, C. Gavoille, A space lower bound for routing in trees, in: Proc. 19th Ann. Symp. Theoret. Aspects Comput. Sci., STACS 2002, in: Lecture Notes in Comput. Sci., vol. 2285, 2002, pp. 65-75.

[13] G.N. Frederickson, R. Janardan, Designing networks with compact routing tables, Algorithmica 3 (1988) $171-190$

[14] C. Gavoille, A survey on interval routing schemes, Theoret. Comput. Sci. 245 (1999) 217-253.

[15] C. Gavoille, N. Hanusse, Compact routing tables for graphs of bounded genus, in: Proc. 26th Int. Colloquium on Automata, Languages and Programming, ICALP 1999, in: Lecture Notes in Comput. Sci., vol. 1644, 1999, pp. 351-360.

[16] C. Gavoille, C. Paul, Optimal distance labeling schemes, in: Proc. 11th Annual European Symp. Algorithms, ESA 2003, in: Lecture Notes in Comput. Sci., vol. 2832, 2003, pp. 254-265.

[17] C. Gavoille, D. Peleg, S. Pérennes, R. Raz, Distance labeling in graphs, in: Proc. 12th Ann. ACM-SIAM Symp. Discrete Algorithms, SODA 2001, ACM-SIAM, 2001, pp. 210-219. 
[18] D. Harel, R. Tarjan, Fast algorithms for finding nearest common ancestor, SIAM J. Comput. 13 (1984) 338-355.

[19] S. Kannan, M. Naor, S. Rudich, Implicit representation of graphs, in: Proc. 20th Symp. Theory of Computing, STOC 1988, ACM, 1988, pp. 334-343.

[20] M. Katz, N.A. Katz, D. Peleg, Distance labeling schemes for well-separated graph classes, in: Proc. 17th Ann. Symp. Theoret. Aspects of Comput. Sci., STACS 2000, in: Lecture Notes in Comput. Sci., vol. 1770, 2000, pp. 516-528.

[21] J. van Leeuwen, R.B. Tan, Interval routing, The Computer J. 30 (1987) 298-307.

[22] R.C. Lyndon, On Dehn's algorithm, Math. Ann. 166 (1966) 208-228.

[23] R.C. Lyndon, A maximum principle for graphs, J. Combin. Theory 3 (1967) 34-37.

[24] R.C. Lyndon, P.E. Schupp, Combinatorial Group Theory, Springer-Verlag, Berlin, 1977.

[25] L. Narayanan, S. Shende, Partial characterizations of networks supporting shortest path interval labeling schemes, Networks 32 (1998) 103-113.

[26] D. Peleg, Proximity-preserving labeling schemes and their applications, in: Proc. 25th Int. Workshop on Graph-Theoretic Concepts in Computer Science, WG 1999, in: Lecture Notes in Comput. Sci., vol. 1665, 1999, pp. 30-41.

[27] D. Peleg, Informative labeling schemes for graphs, in: Proc. 25th Int. Symp. on Math. Foundations of Computer Science, MFCS 2000, in: Lecture Notes in Comput. Sci., vol. 1893, 2000, pp. 579-588.

[28] D. Peleg, Distributed Computing - A locality-sensitive approach, Society for Industrial and Applied Mathematics (SIAM), Philadelphia, PA, 2000.

[29] C. Prisăcaru, P. Soltan, V. Chepoi, On embedding of planar graphs into hypercubes, Proc. Moldavian Acad. Sci., Ser. Math. 1 (1990) 43-50 (in Russian).

[30] N. Santoro, R. Khatib, Labeling and implicit routing in networks, The Computer J. 28 (1985) 5-8.

[31] J.P. Spinrad, Efficient Graph Representations, Fields Institute Monographs, vol. 19, AMS, 2003.

[32] P. Soltan, D. Zambitski, C. Prisăcaru, Extremal Problems on Graphs and Algorithms of Their Solution, Stiinţa Chişinău, 1973 (in Russian).

[33] M. Thorup, U. Zwick, Compact routing schemes, in: Proc. 13th Ann. ACM Symp. on Par. Alg. and Arch., SPAA 2001, ACM, 2001, pp. 1-10. 